\title{
Theoretical investigation of microstructure evolution and deformation of zirconium under neutron irradiation
}

\author{
A. V. Barashev ${ }^{\mathrm{a}, \mathrm{b}^{*}}$, S. I. Golubov ${ }^{\mathrm{a}}$ and R. E. Stoller ${ }^{\mathrm{a}}$ \\ ${ }^{a}$ Materials Science and Technology Division, ORNL, Oak Ridge, TN 37831- 6138, USA \\ ${ }^{\mathrm{b}}$ Center for Materials Processing, Department of Materials Science and Engineering, University \\ of Tennessee, East Stadium Hall, Knoxville, TN 37996-0750, USA
}

\begin{abstract}
The radiation growth of zirconium is studied using a reaction-diffusion model which takes into account intra-cascade clustering of self-interstitial atoms and one-dimensional diffusion of interstitial clusters. The observed dose dependence of strain rates is accounted for by accumulation of sessile dislocation loops during irradiation. The computational model developed and fitted to available experimental data is applied to study deformation of $\mathrm{Zr}$ single crystals under irradiation up to hundred dpa. The effect of cold work and the reasons for negative prismatic strains and co-existence of vacancy and interstitial loops are elucidated.
\end{abstract}

Key words: radiation growth, hcp metals, neutron irradiation, theory, displacement cascades.

PACS codes: 61.80.Az.; 61.80.Hg., 61.82.Bg.

*Corresponding author. Email address: abarashe@utk.edu (A.V. Barashev). 


\section{Introduction}

The radiation growth ( $R G$ ) of Zr-based materials with the hexagonal close-packed (hcp) crystal lattices is one of the damaging mechanisms which affect safe and economical operation of commercial nuclear reactors.

Experiments have demonstrated that deformation of these materials at temperatures below $\sim 300^{\circ} \mathrm{C}$ is driven by nucleation and growth of dislocation loops on both the prismatic $(a)$ and basal (c) planes. In annealed $\mathrm{Zr}$ crystals, the $\mathrm{RG}$ is characterized by expansion along $a$ axes and contraction along $c$ axis. Radiation growth typically consists of three distinct stages [1]. Stage I exhibits a high strain rate and lasts for $\sim 0.1-1.0$ displacements per atom (dpa). Stage II demonstrates a very low strain rate, often interpreted as strain saturation, and proceeds up to $\sim 3$ dpa. At higher doses, during stage III, usually referred to as the breakaway growth stage, the strain rates increase with increasing dose and reach values as high as $\sim 10^{-3} \mathrm{dpa}^{-1}$. The dose dependence of these rates is debated. Transmission electron microscopy exanimation of irradiated samples revealed formation of interstitial-type prismatic loops with $(\mathbf{1} / \mathbf{3})<\mathbf{1 1} \overline{2} \mathbf{0}>$ Burgers vectors during stages I and II, and vacancy-type $c$ loops during stage III. No basal interstitial-type loops are ever observed.

In cold-worked materials the strain rates are relatively high from the very beginning and no strain saturation occurs [2]. In some cases, both $a$ and $c$ strains have been found to be negative. Moreover, vacancy- and interstitial-type prismatic loops of similar densities and sizes may coexist, which is another intriguing feature of the RG phenomenon. This co-existence violates the well-known loop property: vacancy- and interstitial-type loops of large enough size have almost the same efficiencies for absorption of point defects, hence, cannot grow at the same time. This is the reason why their co-existence is never observed in cubic crystals. To our knowledge, these two observations: the negative $a$ strains and co-existence of the vacancy- and interstitial-type prismatic loops have never been explained in a self-consistent way by any model.

Several theoretical models of RG have been published since the first model by Buckley [3]; these models have been reviewed by Holt [4]. All of them, with one exception discussed below, are based on a simplified implementation of mean-field reaction rate theory in which the primary damage is in the form of Frenkel pairs, i.e. single vacancies and self-interstitial atoms (SIAs), both migrating three-dimensionally (3-D). Such an approach is not consistent with current 
knowledge of the primary damage generated in atomic displacement cascades, where significant defect clustering occurs. In our view, this is the reason why several important observations remained unexplained. For example, the conventional concept of dislocation bias employed by these models suggests that the strains must have opposite signs to those generally observed, i.e. positive/expansion in basal $c$ and negative /contraction in prismatic $a$ directions. This is because the Burgers vector of $c$ dislocations is larger than that of $a$ dislocations, which creates stronger interaction of $c$ dislocations rather than $a$ dislocations with SIAs.

A significant step in understanding RG was made by Woo and Gösele [5] and [6] by introducing anisotropic diffusion of single SIAs. In the diffusion anisotropy difference (DAD) model [6] it is assumed that the vacancy diffusion is isotropic, whereas the SIAs migrate preferentially along the basal planes. This provided an explanation of the contraction of $c$ axes and the crucial role of $c$ loops in the breakaway growth of annealed crystals.

Nevertheless, we argue that the DAD model does not correctly describe RG in neutron-irradiated materials because of the assumption that the primary damage consists of only Frenkel pairs. Both experiments [7] and molecular dynamics (MD) simulations [8,9] show that a large fraction, 20-50\%, of the point defects produced in displacement cascades are found in small clusters. The SIA clusters are highly mobile and migrate one-dimensionally (1-D) along close-packed directions in all crystals including $\mathrm{Zr}$ [8] and [10]. This leads to a mixture of the second-order (for the point defects) and the third-order (for the SIA clusters) reaction kinetics [11], rather than just second order, as in the DAD model. The third-order reaction kinetics of SIA clusters with dislocations arises because the reaction rate is proportional to the product of the cluster concentration and the dislocation density squared. This contrasts with the second-order reaction kinetics between point defects and dislocations, which is proportional to the point defect concentration and dislocation density. In the calculations presented in [6], the DAD bias factor for SIAs was required to be equal to $200 \%$ in order to fit the experimental data. This implies a very high anisotropy of single SIA diffusion, with the ratio of diffusion coefficients parallel and perpendicular to the basal planes $D_{\mathrm{a}} / D_{\mathrm{c}} \approx 10^{2}$. Such a high ratio is not supported by a study using a combination of Monte Carlo and ab initio calculations in [12], where it was estimated to be between three and four at temperatures in the range from $300 \mathrm{~K}$ to $1000 \mathrm{~K}$. Moreover, the anisotropy was found to be higher for vacancies than for SIAs at temperatures below $900 \mathrm{~K}$, so that the basic assumptions of the DAD model seem to be invalid. 
Holt et al. [13] made an attempt to generalize the DAD model by accounting for the cascadeproduced SIA clusters, but assumed the clusters to be immobile, which contradicts the MD simulation results mentioned above. One common failure of all these models is that they do not explain the co-existence of vacancy and SIA loops which is observed in irradiated samples.

Recently we have proposed a reaction-diffusion model of $\mathrm{RG}[14,15]$ which is based on the Production Bias Model (PBM) [16-19]. The PBM represents a significant step in the development of theory of void swelling in materials with cubic lattices. Its predictions are consistent with a broad range of experimental results, and account for such observations as enhanced swelling near grain boundaries, and void-lattice formation, which could not be explained by earlier models. This success is due to inclusion of the cascade production and 1-D migration of SIA clusters into the theory.

The displacement cascades in hcp $\mathrm{Zr}$ are similar to those in cubic crystals; hence the PBM should provide a realistic framework for the behavior of hcp metals. For example, the observations of basal-plane alignment of vacancy loops at low temperature [20, 21] and voids at high temperature [22] in zirconium alloys are analogous to void ordering in cubic metals. Such a similarity gives additional support to the idea that, with certain modifications accounting for the hcp lattice structure, the PBM will be capable of describing RG.

The PBM model $[14,15]$ reproduces all the RG stages observed, including the break-away growth in pre-annealed samples at high irradiation doses. In addition, it accounts for such striking observations as negative strains in prismatic directions and the co-existence of vacancy- and interstitial-type prismatic loops, both of which are unexplainable by any model based on the assumption that Frenkel pairs are the only form of initial damage created by incident particles [15]. It follows from the model that accumulation of vacancy- and interstitial-type dislocation loops with increasing irradiation dose changes the relative fractions of dislocations with different Burgers vectors, which leads to the dose dependence of the radiation growth rates. There is no analytical description of this process, and the development of a computational model is the main aim of the present work.

This paper is organized as follows. In Section 2, the model is described. The model was implemented in a computer code named RIMD-ZR.V1 (Radiation Induced Microstructure and Deformation of Zr, Version 1). In Section 3, selected results obtained using the RIMD-ZR code are presented. A summary is given in Section 4. 


\section{The model}

\subsection{Model formulation and assumptions}

The model was implemented using the following assumptions:

1. Initial microstructure consists of $a$ - and $c$-type edge dislocations; densities of dislocations with the Burgers vectors along different $\mathbf{a}_{1}, \mathbf{a}_{\mathbf{2}}$ and $\mathbf{a}_{\mathbf{3}}$ prismatic directions and lying in the basal (c) plane, $\rho_{j}\left(\boldsymbol{j}=\mathbf{a}_{1}, \mathbf{a}_{2}, \mathbf{a}_{3}, \mathbf{c}\right)$, may be unequal.

2. The primary damage produced in displacement cascades consists of mobile point defects and SIA clusters with the Burgers vectors along $\langle\mathbf{1 1} \overline{\mathbf{2}} \mathbf{0}\rangle$ prismatic directions.

3. The point defects execute 3-D random walk on the lattice.

4. The SIA clusters migrate 1-D along their Burgers vectors, i.e. one of the $\langle\mathbf{1 1} \overline{\mathbf{2}} \mathbf{0}\rangle$ directions, parallel to the basal planes.

5. The basal interstitial-type loops are not formed, which is in agreement with experiments.

6. The SIA clusters interact with dislocations of the same Burgers vector only, while the much weaker interaction with other dislocations is ignored. An analysis supporting this assumption is discussed in detail elsewhere [14,15].

7. The dislocation bias factor for point defects, mutual recombination of point defects, and thermal vacancies are ignored.

The population of edge dislocations and dislocation loops, which evolve during irradiation, is characterized by the total dislocation length per unit volume for each Burgers vector $\rho_{i}\left(\boldsymbol{j}=\mathbf{a}_{1}, \mathbf{a}_{2}, \mathbf{a}_{3}, \mathbf{c}\right)$, thus neglecting the difference in the absorption properties of loops and dislocations with both point defects and SIA clusters:

$$
\rho_{j}=\rho_{\mathrm{d}}^{j}+2 \pi r_{\mathrm{v}}^{j} N_{\mathrm{v}}^{j}+2 \pi r_{\mathrm{i}}^{j} N_{\mathrm{i}}^{j}
$$

where $\rho_{\mathrm{d}}^{j}$ is the edge dislocation density, and $\boldsymbol{r}_{\mathrm{v}, \mathrm{i}}^{j}$ and $\boldsymbol{N}_{\mathrm{v}, \mathrm{i}}^{j}$ are the mean radius and number density of vacancy (subscript v) and interstitial (subscript i) type loops of $j$ orientation of the Burgers vector.

\subsection{Concentrations of mobile defects}


The system evolves due to reactions involving mobile defects, which cause dislocation climb, and sessile loops grow or shrink. The concentrations, $C$, of mobile defects: single vacancies (subscript v), single SIAs (i) and SIA clusters (cl) are obtained from the steady-state balance equations ( $\left.m=a_{1}, a_{2}, a_{3}\right)$ :

$$
\begin{gathered}
\bigotimes_{\mathrm{v}}=G-\rho D_{\mathrm{v}} C_{\mathrm{v}}=0, \\
\bigotimes_{\mathrm{i}}=G\left(1-\varepsilon_{\mathrm{i}}^{\mathrm{g}}\right)-\rho D_{\mathrm{i}} C_{\mathrm{i}}=0, \\
\bigotimes_{\mathrm{cl}}^{m}=G \frac{\varepsilon_{\mathrm{i}}^{\mathrm{g}}}{3 r^{p}}-k_{m}^{2} D_{\mathrm{cl}} C_{\mathrm{cl}}^{m}=0,
\end{gathered}
$$

where $\boldsymbol{G}=\boldsymbol{G}^{\mathrm{NRT}}\left(\mathbf{1}-\varepsilon_{\mathrm{r}}\right)$ is the point defect production rate in dpa per second, $\boldsymbol{G}^{\mathrm{NRT}}$ being the NRT standard dose rate $[\mathrm{dpa} / \mathrm{s}], \varepsilon_{\mathrm{r}}$ the fraction of defects recombining in the cooling down stage of cascades and $\varepsilon_{\mathrm{i}}^{\mathrm{g}}$ the fraction of SIAs produced in cascades in the form of 1-D mobile clusters; $\boldsymbol{D}_{\mathrm{v}}$, $D_{\mathrm{i}}$ and $\boldsymbol{D}_{\mathrm{c}}$ are the diffusion coefficients of vacancies, SIAs and SIA clusters, respectively, $\eta_{\mathfrak{l}}$ is the mean number of SIAs in a cascade-produced mobile cluster; $\rho=\sum_{j} \rho_{j}\left(j=\mathbf{a}_{1}, \mathbf{a}_{2}, \mathbf{a}_{3}, \mathbf{c}\right)$ is the total density of edge dislocations and dislocation loops for all orientations of the Burgers vector. The sink strength for the SIA clusters migrating along $m$ direction, $\boldsymbol{k}_{\boldsymbol{m}}^{2}$, is given by $[14,19]$ :

$$
k_{m}^{2}=2\left(\frac{\pi r_{\mathrm{cd}} \rho_{m}}{2}\right)^{2}
$$

where $r_{c d}$ is the capture radius of dislocations and loops for mobile SIA clusters. The first term in the right-hand sides (RHSs) of Eqs. (2)-(4) account for the production of point defects and SIA clusters, while the second term for their loss at dislocations, including sessile loops. The factor $1 / 3$ in Eq. (4) accounts for the equality of SIA cluster production rates in all three prismatic directions.

\subsection{Growth/Shrinkage of sessile dislocation loops}


To describe the growth/shrinkage of sessile dislocation loops we use the mean-size approximation, where the mean values of radii, $\boldsymbol{r}_{\mathrm{v}, \mathrm{i}}^{j}$, for sessile vacancy and interstitial loops are found via the relationship between the loop number density, $N\left[\mathrm{~m}^{-3}\right]$, the total number of defects in the loops of any particular type, $S$ [per lattice site], and the loop Burgers vector $\boldsymbol{b}$ :

$$
S=\pi r^{2} b N
$$

Eq. (6) is approximate, because the exact equation is proportional to $\left\langle r^{2}\right\rangle$ (mean value of the radius squared) not $\langle\boldsymbol{r}\rangle^{2}$ (which, here and below is denoted as $\boldsymbol{r}^{2}$ ), which are only the same if the dispersion of the loop radii (the width of the size distribution) is zero. The values $N$ and $S$ are found from 'exact' equations (exact in a sense that its form is independent of whether the mean-field approximation was used), which do not require knowledge of the loop size distributions. The total numbers of defects (per atomic site) in the loops change with time due to reactions with point defects and SIA clusters according to the following equations ( $\left.m=a_{1}, a_{2}, a_{3}\right)$.

$$
\begin{gathered}
\xi_{\mathrm{v}}^{m}=2 \pi r_{\mathrm{v}}^{m} N_{\mathrm{v}}^{m}\left(D_{\mathrm{v}} C_{\mathrm{v}}-D_{\mathrm{i}} C_{\mathrm{i}}\right)-k_{v m}^{2} \eta^{\rho} D_{\mathrm{cl}} C_{\mathrm{cl}}^{m}, \\
\xi_{\mathrm{i}}^{m}=-2 \pi r_{\mathrm{i}}^{m} N_{\mathrm{i}}^{m}\left(D_{\mathrm{v}} C_{\mathrm{v}}-D_{\mathrm{i}} C_{\mathrm{i}}\right)+k_{l m}^{2} \eta^{\dagger} D_{\mathrm{cl}} C_{\mathrm{c}}^{m}, \\
\xi_{\mathrm{v}}^{m}=2 \pi r_{\mathrm{v}} N_{\mathrm{v}}\left(D_{\mathrm{v}} C_{\mathrm{v}}-D_{\mathrm{i}} C_{\mathrm{i}}\right) .
\end{gathered}
$$

In these equations we assume that the defect absorption rate by loops of any size is proportional to the loop circumference (such an assumption has already been introduced in section 2.1). Such a description may fail for very small loops, where the proportionality to the loop area may be a better approximation. But such a simplification should not influence the main trends of the model. Note that the analysis depends on the total interstitial flux $\boldsymbol{n}_{\mathfrak{q}} \boldsymbol{D}_{\mathrm{cl}} C_{\mathrm{cl}}^{m}$, and since $C_{\mathrm{cl}}^{m} \propto\left(\boldsymbol{n}^{\mathfrak{p}}\right)^{-\mathbf{1}}$, the flux is independent of $\boldsymbol{\eta}_{\mathfrak{f}}$. The partial sink strengths of sessile vacancy and interstitial loops for mobile SIA clusters in these equations are given by $[14,19]$ ( $\left.k=\mathbf{i}, \mathbf{v}, \boldsymbol{m}=\mathbf{a}_{1}, \mathbf{a}_{2}, \mathbf{a}_{3}\right)$

$$
k_{k m}^{2}=\left(\pi r_{c d}\right)^{2}\left(\pi r_{k}^{m} N_{k}^{m}\right) \rho_{m}
$$


We emphasize that Eq. (10) accounts for the interaction of SIA clusters with dislocations of the same Burgers vector only, which is one of the main assumptions of the present model, as stated in section 2.1 .

By substituting concentrations of the mobile defects from Eqs. (2)-(4) and Eq. (6) into the RHS and LHS of Eqs. (7)-(9), respectively, one obtains the following equations for the time evolution of the mean loop radii $\left(\boldsymbol{m}=\mathbf{a}_{1}, \mathrm{a}_{2}, \mathrm{a}_{3}\right)$ :

$$
\begin{gathered}
\frac{d e^{m}}{d \phi}=\frac{\chi}{b^{2}}\left(\frac{1}{\varrho}-\frac{1}{3 \rho_{m}}\right) \\
\frac{d p^{m}}{d \phi}=-\frac{\chi}{b^{2}}\left(\frac{1}{\varrho}-\frac{1}{3 \rho_{m}}\right) \\
\frac{d f}{d \phi}=-\frac{\chi}{b \rho},
\end{gathered}
$$

where $\phi=\boldsymbol{G}^{\mathrm{NRT}} \boldsymbol{t}$ is the irradiation dose in dpa, $\boldsymbol{b}^{\boldsymbol{a}}$ and $\boldsymbol{b}$ are the Burgers vectors of prismatic and basal dislocations, respectively, and $\chi=\left(1-\varepsilon_{\mathrm{r}}\right) \varepsilon_{\mathrm{i}}^{\mathrm{g}}$. The value $\chi$ is the main parameter of the model; its physical significance is the fraction of the total number of displaced atoms that are produced in the form of 1-D mobile interstitial clusters.

\subsection{Growth strains}

To formulate equations for the strain rates, let us first consider the change of the quantities $E$ : the net numbers of SIAs (i.e. the number of SIAs minus number of vacancies) accumulated by dislocations and loops in different directions. It follows from Eqs. (2)-(4) that these are equal to the sums of the contributions from glissile SIA clusters (for prismatic directions), and point defects captured by dislocations and sessile loops (for all directions) $\left(\boldsymbol{m}=\mathbf{a}_{1}, \mathbf{a}_{\mathbf{2}}, \mathbf{a}_{\mathbf{3}}\right)$ :

$$
\begin{aligned}
& \Sigma_{m}^{\&}=\frac{1}{3} \chi \boldsymbol{G}^{\mathrm{NRT}}-\rho_{m}\left(D_{\mathrm{v}} C_{\mathrm{v}}-D_{\mathrm{i}} C_{\mathrm{i}}\right)+\sum_{m}^{\text {Rucl }}, \\
& \sum_{\mathrm{c}}^{\&}=-\rho_{\mathrm{c}}\left(\boldsymbol{D}_{\mathrm{v}} \boldsymbol{C}_{\mathrm{v}}-\boldsymbol{D}_{\mathrm{i}} \boldsymbol{C}_{\mathrm{i}}\right)+\boldsymbol{E}_{\mathrm{c}}^{\text {Rucl }} \text {. }
\end{aligned}
$$


In these equations, the SIA cluster contribution to strains is accounted for by the first term on the RHS of Eq. (14), the \&ucl are due to nucleation of sessile loops, while the other terms are due to collisions of dislocations and sessile loops with the point defects. Our numerical analysis in the following sections showed that in order to reproduce experimental observations, the fraction of SIAs produced as sessile loop nuclei make a negligible contribution to strains as compared to the other terms, i.e. the $\mathbb{R}^{\text {nucl }}$ are negligible.

Below the Cartesian coordinate system with the $x$ and $y$ axes lying in the basal plane, where the $x$ axis makes an angle $a$ with $\mathbf{a}_{\mathbf{1}}$ direction (Fig. 1) is used. Generally the strains are described by the six independent components of strain tensor. In the Appendix, the coordinate systems in which the strain tensor is diagonal are found. It is conclude that for $\alpha=\mathbf{0}$, i.e. when $x$ is along $\mathbf{a}_{1}, y$ along $\mathbf{a}_{3}-\mathbf{a}_{2}$ and $z$ along $\mathbf{c}$ directions, the strains along principal axes are correctly described by the following equations:

$$
\begin{gathered}
\varepsilon_{\mathrm{x}}=E_{\mathrm{a}_{1}}+\left(E_{\mathrm{a}_{2}}+E_{\mathrm{a}_{3}}\right) \cos ^{2}(\pi / 3), \\
\varepsilon_{\mathrm{y}}=\left(E_{\mathrm{a}_{2}}+E_{\mathrm{a}_{3}}\right) \sin ^{2}(\pi / 3), \\
\varepsilon_{\mathrm{z}}=E_{\mathrm{c}^{\prime}}
\end{gathered}
$$

By substituting Eq. (14)-(15) into the time derivative of Eqs. (16)-(18), where the nucleation terms are omitted, then, with the aid of Eqs. (2)-(4), one can obtain the following equations for the diagonal elements of the strain tensor, $\varepsilon_{j} \equiv \varepsilon_{j \boldsymbol{j}},(\boldsymbol{i}=\mathbf{x}, \mathbf{y})$ :

$$
\begin{gathered}
\left.\frac{d \varepsilon_{i}}{d \phi}=\chi \frac{(1}{2}-\frac{\rho_{i}}{\rho}\right) \\
\frac{d \varepsilon_{\mathrm{z}}}{\boldsymbol{d} \phi}=-\chi \frac{\rho_{\mathrm{z}}}{\rho}
\end{gathered}
$$

where the effective dislocation densities in corresponding directions are defined as

$$
\begin{gathered}
\rho_{\mathrm{x}}=\rho_{\mathrm{a}_{1}}+\left(\rho_{\mathrm{a}_{2}}+\rho_{\mathrm{a}_{3}}\right) \cos ^{2}(\pi / 3), \\
\rho_{\mathrm{y}}=\left(\rho_{\mathrm{a}_{2}}+\rho_{\mathrm{a}_{3}}\right) \sin ^{2}(\pi / 3),
\end{gathered}
$$




$$
\rho_{\mathrm{z}}=\rho_{\mathrm{c}}
$$

which conserve the total density: $\rho_{\mathrm{x}}+\rho_{\mathrm{y}}=\rho_{\mathrm{a}_{1}}+\rho_{\mathrm{a}_{2}}+\rho_{\mathrm{a}_{3}}$. Eqs (19)-(20) are the main outcome of references [14] and [15].

\subsection{Nucleation of sessile loops}

The equation system formulated above allows the calculation of the growth/shrinkage of vacancy and interstitial loops and dislocation climb, as well as the resulting strains. It does not include, however, the evolution of the loop number densities $\mathbf{N}_{\boldsymbol{k}}^{j}(\boldsymbol{k}=\mathbf{v}, \mathbf{i})$ for different $j$ Burgers vector directions. Thus, to describe the dose dependence, a model for loop nucleation is required. A comprehensive description of the evolution of the population of sessile vacancy- and interstitialtype dislocation loop size distributions can be obtained from the cluster dynamics master equations in which describe the reaction rates of mobile defect species with the loops. This generally requires a large number of equations, one for each cluster size [23]. In the absence of all the fundamental rate coefficients the complete master equation approach would require, we use a simplified description for the nucleation process, which reproduces qualitatively the experimental observations, and the mean-size approximation for loop growth.

Not much is known about the nucleation of loops. Available experimental data can be summarized as follows: (a) $a$ loops nucleate from the very beginning of irradiation and reach a typical density of $\sim 10^{22} \mathrm{~m}^{-3}$ after several dpa, and (b) $c$ loops are of vacancy type and do not appear to nucleate prior to a dose of $\sim 3 \mathrm{dpa}$ and reach an order of magnitude smaller density, $\sim 10^{21} \mathrm{~m}^{-3}$ (see [24,25] for reviews and [26,27] for observations). The reasons for the incubation dose for nucleation of basal vacancy loops are unknown as yet. A more detailed treatment of $a$ and $c$ loop nucleation mechanisms, including temperature dependence of the final loop densities, is beyond the scope of the present work. In this paper, the following description is used, which reproduces the experimental observations mentioned above:

1. Interstitial and vacancy prismatic loops are formed at constant rate from the very beginning of irradiation, until the maximum number densities, $\mathbf{N}_{\mathbf{v}, \mathbf{i}}^{a, \max }$, the values of which may depend on irradiation conditions and are reached at a dose $\phi_{\max }^{\mathrm{a}}$, chosen to fit the observed strain behavior. 
2. Basal vacancy loops nucleate after some critical dose, $\phi_{\mathbf{0}}^{\mathbf{c}}$, until reaching a maximum number density, $\boldsymbol{N}_{\mathbf{v}}^{\mathbf{c}, \max }$. The nucleation rate is more complicated in this case (Eq. (25)), and its functional dependence was chosen to reproduce the observed strain behavior at the beginning of the break-away stage.

3. The nucleation of vacancy- or interstitial-type sessile loops takes place if the net flux of corresponding defects, vacancies or SIAs, is positive.

To describe evolution of the loop number densities, we use the following equations.

$$
\begin{aligned}
& \frac{d N_{. i}^{m}}{d \phi}=\left\{\begin{array}{ll}
N_{v . i}^{a \max } / 3 \phi_{\max }^{\mathrm{a}}, & N_{\mathrm{v} . \mathrm{i}}^{m} \leq N_{\mathrm{v} . \mathrm{i}}^{\mathrm{a} \max } / 3, \\
0, & N_{\mathrm{v} . \mathrm{i}}^{m} \geq N_{\mathrm{v} . \mathrm{i}}^{a, m a x} / 3,
\end{array} \quad m=\mathrm{a}_{1}, \mathrm{a}_{2}, \mathrm{a}_{3},\right. \\
& \frac{d N}{d \phi}= \begin{cases}0, & \phi<\phi_{0}^{c}, \\
N_{v}^{c \max } \frac{A \exp \left[A\left(\phi-\phi_{0}^{\mathrm{c}}\right) /\left(\phi_{\max }^{\mathrm{c}}-\phi_{0}^{\mathrm{c}}\right)\right]}{\left.\left(\phi_{\max }^{\mathrm{c}}-\phi_{0}^{\mathrm{c}}\right) \exp (A)-1\right]}, & \phi \geq \phi_{0}^{\mathrm{c}}, \boldsymbol{N}_{\mathrm{v}}^{\mathrm{c}}<N_{\mathrm{v}}^{\mathrm{c}, \max }, \\
0, & \phi \geq \phi_{0}^{\mathrm{c}}, \boldsymbol{N}_{\mathrm{v}}^{\mathrm{c}} \geq \boldsymbol{N}_{\mathrm{v}}^{\mathrm{c} \text { max }} .\end{cases}
\end{aligned}
$$

In these equations, $A$ is a dimensionless parameter for fitting the dose dependence of the nucleation rate of basal vacancy loops; small values of $A$ correspond to a linear increase in the nucleation rate with increasing dose, whereas high values lead to a steep exponential increase. For any $A$, the integral of $\boldsymbol{d} \boldsymbol{N} / \boldsymbol{d}$ over dose from $\phi_{0}^{\mathrm{c}}$ to $\phi_{\max }^{\mathrm{c}}$ gives $\boldsymbol{N}_{\mathbf{v}}^{c, m a x}$. Since there is an incubation dose, $\phi_{0}^{c}$, for $c$ loop nucleation, we expect that the nucleation rate may further increase during irradiation, and this is the reason for such a complicated description. Obviously, the terminal dose for nucleation of the basal vacancy-type loops, $\phi_{\max }^{c}$, and the maximum loop density, $\boldsymbol{N}_{\mathbf{v}}^{\text {c, } \max }$, are interdependent; one can fix either of them and the other will be defined. We use the measured number density as the independent parameter and find a corresponding terminal dose. Similarly, $\phi_{\max }^{\mathrm{a}}$ is the parameter which defines the nucleation rate of both vacancy- and interstitial-type prismatic loops. It is the terminal dose for nucleation of prismatic interstitial-type loops for an isotropic distribution of prismatic dislocations, when no prismatic vacancy loops are formed.

Fig. 2 shows the evolution of the number densities of interstitial-type prismatic loops and vacancy-type basal loops, calculated using Eqs. (24) and (25) with the parameters presented in 
Table 1. The total number density in this figure is the sum of densities of prismatic loops with different Burgers vectors: $\boldsymbol{N}^{\mathrm{a}, \max }=\boldsymbol{N}^{\mathrm{a} 1, \max }+\boldsymbol{N}^{\mathrm{a} 2, \max }+\boldsymbol{N}^{\mathrm{a}, \text { ma }}$. These functions are similar to the experimental data in [24] and are used as the basic parameter set for the calculations presented below.

\section{Calculations}

A computer code RIMD-ZR.V1 (Radiation Induced Microstructure and Deformation of Zr, Version 1) implementing the scheme described above has been developed and used to study evolution of the microstructure during neutron irradiation. Selected results are presented below.

\subsection{Fitting of the model to experimental data}

It should be emphasized that we do not aim to exactly reproduce any particular experiment in this paper. If this were the case, a more detail information on the material microstructure, e,g, the anisotropy of the dislocation density distribution, would be required. Instead, we aim to demonstrate the capability of the model to reproduce the observed positive and negative strains and coexistence of vacancy- and interstitial-type loops by using a physically sound set of input parameters, including reasonable description of the observed defect densities.

Fig. 3 shows the results of using experimental data from [24] to adjust model parameters, which are collected in Table 1 and presented in Fig. 2. Note that the best fit parameters $\varepsilon_{\mathrm{r}}=0.9$ and $\varepsilon_{\mathrm{i}}^{\mathrm{g}}$ $=0.2$ correspond to $x=0.02$, which coincides with that extracted from experiments on fcc $\mathrm{Cu}$ in [16]. The experimental data on $a$ and $c$ strains (actual measurements, not extrapolated data, as in Fig 4 b in [24]) are shown on Figs. 3 and 4, but only $a$ strains were used in fitting.

The results in Fig. 3 were obtained for single crystal and an isotropic distribution of prismatic dislocations. The theoretical $c$ strain in this case is two times larger in absolute value than that along any of the $a$ directions. This is not always the case in experiments, as shown, e.g. in Fig.3 and 4 here (see also Fig. 3a of [24]), where the $c$ strain is significantly smaller than $a$ strain. (Note that the measurements were made on two different $a$ - and $c$-axis crystals.) Thus, the proposed model underestimates the observed $c$ strains, and one of the reasons for this discrepancy may be a non-isotropic distribution of prismatic dislocations, which lead to negative strains in one of the $a$ direction. The latter effect is considered in the following sections, see the 
text related to Figs. 13, 14 and 16. Indeed, since the sum of $x, y$ and $z$ strains is equal to zero in the absence of swelling, the absolute value of $z$ strain may be significantly smaller than $x, y$ strains if $x$ and $y$ strains have opposite signs.

The strain behavior calculated for a wider dose range, which includes the beginning of the breakaway stage, is presented in Fig. 4 for the same parameters as in Fig. 3. These results demonstrate that the function chosen to describe nucleation of $c$-type vacancy loops gives prismatic strain values during the break-away stage which are similar to those observed experimentally (e.g., Fig. 4a in [24]). Note that the $c$ strains for irradiation doses higher than 3.5dpa have not been measured in [24], only extrapolations made on the basis of some assumptions. This is why we have only prismatic strains to compare with.

Fig. 5 shows the dose dependence of interstitial and vacancy loops radii for the same parameter set. The calculated values are similar to the observed $\sim 10 \mathrm{~nm}$ for $a$-type interstitial loops (e.g., Fig. 3 in [28]) and much larger vacancy $c$ loops. Note that the small decrease in the mean size of $a$ loops in the intermediated dose range may be an artificial effect due to the mean-size approximation used. However, this is not a problem since the more important integral values, which affect strain behavior, behave well; e.g. the loop sink strength increases steadily with increasing irradiation dose, as shown in Fig. 6.

\subsection{Effect of cold work}

The effect of cold-work on the strain behavior is demonstrated in Fig. 7. For all calculations but one, the ratio of $a$ to $c$ dislocation densities was $\rho_{\mathbf{d}}^{\mathbf{a}} / \rho_{\mathbf{d}}^{\mathbf{c}}=5$, as indicated. An increase of all dislocation densities by an order of magnitude increases the strains (dashed line, cf. with solid line calculated with the basic parameter set), but the tendency toward strain saturation is maintained. Another tenfold (or higher) increase of the densities gives steep straight (dotted) line with the slope $\sim 1.7 \times 10^{-3} \mathrm{dpa}^{-1}$, as shown in the figure. This is in a good agreement with the maximum value of $\sim 10^{-3} \mathrm{dpa}^{-1}$ observed for swaged $\mathrm{Zr}$ crystals in [24]. The value of $\sim 10^{-3} \mathrm{dpa}^{-1}$ is reproduced by our calculations when the dislocation density is increased to $10^{15} \mathrm{~m}^{-2}$ and if the ratio of $a$ to $c$ dislocation densities increased to $\rho_{\mathbf{d}}^{\mathrm{a}} / \rho_{\mathbf{d}}^{\mathrm{c}}=\mathbf{1 0}$ (dash-dotted line).

\subsection{Radiation growth at high doses}


Fig. 8 shows the dose dependence of growth strain at irradiation doses of up to $100 \mathrm{dpa}$. Two calculations, for low and high dislocation densities, are presented. As shown in Fig. 7, this difference leads to remarkable qualitative, as well as quantitative, differences in the strain behavior in the dose range up to $2.5 \mathrm{dpa}$ (cf. solid and dotted lines). The difference between the predicted strains diminishes at high doses. This is because the sink strength of sessile loops becomes significantly higher than that of pre-existing dislocations very early during irradiation due to nucleation and growth of sessile loops. This is illustrated in Figs. 9 and 10, which show the dose dependence of the loop radii and the loop sink strength, respectively. The sink strength of these loops thus governs the strain behavior at high enough irradiation doses, which is then independent of initial dislocation density. In the high-dose range, the calculations presented in Fig. 8 depend mainly on the ratio of number densities of sessile $a$-type interstitial and $c$-type vacancy loops, $\boldsymbol{N}_{\mathrm{i}}^{\mathrm{a}, \max } / \boldsymbol{N}_{\mathrm{v}}^{\mathrm{c}, \max }$. A value of 10 was used in these calculations, as indicated in the figure.

The effect of increasing the ratio of $a$ - to $c$-loop densities is shown in Fig. 11. It follows from this calculation that suppressing the nucleation of $c$-type loops decreases the strains and may thereby enhance the radiation resistance of $\mathrm{Zr}$.

These results indicate that at relatively high irradiation doses, the dislocation loops govern the strain behavior. In this case, one can derive an equation for the strain rate in terms of dislocation loop number densities as follows. In the case considered, the number of vacancies in the $c$-type vacancy loops is approximately equal to the number of SIAs in the $a$-type interstitial loops: $\pi\left(\boldsymbol{r}_{\mathrm{i}}^{\mathrm{a}}\right)^{2} \boldsymbol{N}_{\mathrm{i}}^{\mathrm{a}, \max } \boldsymbol{b}=\pi\left(\boldsymbol{r}_{\mathrm{v}}^{c}\right)^{2} \boldsymbol{N}_{\mathrm{v}}^{\mathrm{c}, \max } \boldsymbol{b}$, and it can be readily obtained from Eqs. (19)-(20) and (1) that

$$
\frac{d \varepsilon_{\mathrm{z}}}{d \phi}=-2 \frac{d \varepsilon_{x}}{d \phi}=-2 \frac{d \varepsilon_{y}}{d \phi}=-\chi \frac{1}{1+\sqrt{N_{\mathrm{i}}^{a, \max } / N_{\mathrm{v}}^{c, \max }}}
$$

According to this equation, if the number density of $c$-type vacancy loops is low as compared to interstitial prismatic loops: $\boldsymbol{N}_{\mathbf{i}}^{\text {a,max }} \gg \boldsymbol{N}_{\mathbf{v}}^{\text {cmax }}$, the strain rate is proportional to the square root of the ratio of the loop number densities:

$$
\left(\frac{d \varepsilon_{\mathrm{z}}}{(d \phi}\right)_{\phi \rightarrow \infty} \approx-\chi \sqrt{\frac{N_{\mathrm{v}}^{\mathrm{c}^{\mathrm{max}}}}{N_{\mathrm{i}}^{a, \max }}}
$$


Note that since the nucleation period is limited in time, the asymptotic values of the strain rates can be predicted at relatively low doses, when the loop number densities reach steady state.

\subsection{Effect of vacancy loops on radiation growth}

It can readily be shown using Eqs. (11)-(12) that for the situation in which the total dislocation density in one direction (e.g. $\mathbf{a}_{1}$ ) is higher than in the other two directions, the vacancy loops grow in this direction, while interstitial loops grow in the other two directions. For this case, the RHS of Eq. (11) must be positive, i.e.

$$
2 \rho_{\mathrm{a}_{1}}-\rho_{\mathrm{a}_{2}}-\rho_{\mathrm{a}_{3}}>\rho_{\mathrm{c}} \text {. }
$$

This condition may be true at the beginning of the irradiation, when only pre-existing dislocations are present. However, if interstitial $a$-loop line density accumulates faster than that

of a-type vacancy loops, then $\rho_{\mathrm{a}_{2}}+\rho_{\mathrm{a}_{3}}$ will increase faster than $\rho_{\mathrm{a}_{1}}$ and the condition defined by Eq. (28) will eventually be broken, the RHS of Eq. (11) will become negative, and the vacancy loops will start to shrink. The condition for the latter scenario can be obtained considering $\boldsymbol{d} \rho=\mathbf{2} \pi \boldsymbol{N} \boldsymbol{d} \iota$ and using Eqs. (11)-(12) for the radius change. For simplicity we assume that the dislocation densities and loop parameters in $\mathbf{a}_{2}$ and $\mathbf{a}_{3}$ directions are the same. In this case, one can obtain that

$$
\begin{gathered}
\frac{\boldsymbol{d} \rho_{\mathrm{a}_{1}}}{\boldsymbol{d} \phi}=\chi \frac{2 \pi \boldsymbol{N}_{\mathrm{v}}^{\mathrm{a}_{1}}}{3 \boldsymbol{b}}\left[\frac{2\left(\rho_{\mathrm{a}_{1}}-\rho_{\mathrm{a}_{2}}\right)-\rho_{\mathrm{c}}}{\rho_{\mathrm{a}_{1}} \rho}\right] \\
\frac{\boldsymbol{d} \rho_{\mathrm{a}_{2}}}{\boldsymbol{d} \phi} \equiv \frac{\boldsymbol{d} \rho_{\mathrm{a}_{3}}}{\boldsymbol{d} \phi}=\chi \frac{2 \pi \boldsymbol{N}_{\mathrm{i}}^{\mathrm{a}_{2}}}{3 \boldsymbol{b}}\left[\frac{\left(\rho_{\mathrm{a}_{1}}-\rho_{\mathrm{a}_{2}}\right)+\rho_{\mathrm{c}}}{\rho_{\mathrm{a}_{2}} \rho}\right] .
\end{gathered}
$$

The line density of interstitial loops increases faster if the ratio of these equations is smaller than unity:

$$
\frac{\beta_{\mathrm{a}_{1}}}{\boldsymbol{\beta}_{\mathrm{a}_{2}}}=\frac{2 N_{\mathrm{v}}^{\mathrm{a}_{1}} \rho_{\mathrm{a}_{2}}}{N_{\mathrm{i}}^{\mathrm{a}_{2}} \rho_{\mathrm{a}_{1}}}\left[1-\frac{3}{2} \frac{\rho_{\mathrm{c}}}{\left(\rho_{\mathrm{a}_{1}}-\rho_{\mathrm{a}_{2}}\right)+\rho_{\mathrm{c}}}\right]<\mathbf{1}
$$


This scenario is considered in Figs. 12 and 13, which were calculated with $\boldsymbol{N}_{\mathrm{v}}^{\mathbf{a}_{1}}=\boldsymbol{N}_{\mathrm{i}}^{\mathrm{a}_{2}}$ and $\rho_{\mathbf{d}}^{\mathrm{a}_{1}}=6 \rho_{\mathbf{d}}^{\mathrm{a}_{2}}$ (see inset in Fig. 13). The vacancy loops in $\mathbf{a}_{1}$ direction and interstitial loops in $\mathbf{a}_{2}$ and $\mathbf{a}_{3}$ directions grow very fast for a short period. Then, vacancy loops start to dissolve, and interstitial loops start to grow in $\mathbf{a}_{1}$ direction. Vacancy loops in $\mathbf{a}_{2}$ and $\mathbf{a}_{3}$ directions grow temporarily, then shrink during a transition period, when vacancy loops shrink in $\mathbf{a}_{1}$ direction. Then, interstitial-type loops grow in all three $a$ directions.

Fig. 13 shows that for the case considered, the strain along the direction where vacancy loops grow is negative for about six dpa. Then, after vacancy loops dissolve, the strain becomes positive and increases at a rate similar to the other two directions. This picture is similar to that observed experimentally in [24]. At higher irradiation doses the $x$ and $y$ strains approach each other, as shown by black dotted and dashed lines in Fig. 14.

Fig. 14 shows the dose dependence of the growth strain for the same case as in Fig. 13, designated as low dislocation density, and for the case when all dislocation densities are increased by an order of magnitude, designated as high dislocation density. The loop radii for the higher dislocation density are shown in Fig. 15. For the higher dislocation density, vacancy loops grow over a longer dose range, but the accumulation of loops eventually breaks the inequality of Eq. (28). Then, the $x$ strain changes its sign and approaches the asymptotic behavior of the $y$ strain. Thus, the observed negative $a$ strains, which have been discussed extensively in the literature (see, e.g. [20]), are explained by the present calculations and are physically related with the transitory formation followed by disappearance of prismatic vacancy loops in conditions of anisotropic dislocation distribution.. Moreover, it is shown that the negative strains are expected to exist only in a certain dose range and disappear at high enough doses.

In the calculations discussed above, vacancy loops grow in one $a$ direction and interstitial loops in the other two $a$ directions, which is due to corresponding distribution of initial dislocation densities in these directions. The situation is symmetric; vacancy loops may grow in two directions and interstitial loops in one direction if the dislocation densities in two $a$ directions are higher. This is demonstrated in Figs. 16 and 17 for the loop strains and radii, respectively. The description of the Figs. 16 and 17 is the same as for Figs. 14 and 15, and the scenario in which vacancy loops initially grow but then dissolve is realized in this case as well. 
The results of this section shed light on the observation stated. by Holt [1] that "the important new experimental observations which influence our perception of the growth phenomenon in zirconium alloys include ... the occurrence of "negative" growth, i.e., contractions along prism directions...". Experiments showed that specimens of single-crystal Zr which were strained 5\% in the prism direction, exhibited negative prismatic strain which decreased with time [2]. Asreceived polycrystalline $\mathrm{Zr}$ also showed negative prismatic strains, which increased for about one dpa and then changed sign [2], a behavior which is similar to the behavior analyzed in this section.

\section{Summary}

1. A model of the dose dependence of radiation growth of in zirconium and Zr-alloys has been formulated.

2. The computer code RIMD-ZR.V1 (Radiation Induced Microstructure and Deformation of $\mathrm{Zr}$, Version 1), which implements the model of radiation growth in $\mathrm{Zr}$ proposed in [14] and [15], and uses the mean-size approximation for the description of sessile vacancy and interstitial loops, has been developed.

3. The basic parameters of the model (Table 1), namely the fractions of defects recombining and clustering in cascades have been fitted to the experimental data available and found to be the same as those extracted from swelling experiments on fcc copper in [16], and those required to reproduce steady-state swelling rates observed in austenitic stainless steels at high irradiation doses [17].

4. The calculations reproduce the observed growth stages, including the 'break-away' growth of pre-annealed materials, the negative strains in prismatic directions and coexistence of vacancy- and interstitial-type prismatic loops.

5. The strain rates at high enough doses are determined by the ratio of number densities of interstitial prismatic and vacancy basal loops, Eq. (26). These values may be predicted at relatively small doses, when the nucleation stage is completed.

6. The calculations confirm the maximum strain rate of $\sim 1 \% \mathrm{dpa}^{-1}$ predicted by the model [14] and [15]. This may occur for a highly anisotropic distribution of prismatic dislocations. It requires that the vacancy and interstitial prismatic loops co-exist, so that the crystal expands in one of $a$ directions and contracts in the other, with relatively small $c$ strain. 
The calculations have revealed a crucial dependence of the strains on the loop number densities, especially in the high dose limit, Eq. (26). Hence, further progress in this area is only possible if the physical mechanisms of sessile loop nucleation are elucidated.

Finally, we would like to emphasize that the predicted maximum value of the $\mathrm{RG}$ strain rate for $\mathrm{Zr}$ of $\sim 1 \% \mathrm{dpa}^{-1}$ is the same as the maximum swelling rate found in cubic materials [17]. This follows from the similarity of cascade-induced radiation damage in the bcc, fcc and hcp metals, and indicates that the Production Bias Model [19], which was originally developed for cubic materials, provides a general framework for the theory of radiation damage.

\section{Acknowledgements}

This research was supported by the Consortium for Advanced Simulation of Light Water

Reactors (http://www.casl.gov), an Energy Innovation Hub (http://www.energy.gov/hubs) for Modeling and Simulation of Nuclear Reactors under U.S. Department of Energy Contract No. DE-AC05-00OR22725. 


\section{Appendix}

Generally, the strains in prismatic directions are described by a two-dimensional tensor, $\varepsilon_{i k}$, which can be found using Eq. (14). Using simple geometrical consideration, one can obtain that, in a Cartesian coordinate system with the $x$ axis lying in the basal plane and making an angle $a$ with $\mathbf{a}_{1}$ direction (see Fig. 1):

$$
\begin{gathered}
\varepsilon_{\mathrm{x}}=E_{\mathrm{a}_{1}} \cos ^{2} \alpha+E_{\mathrm{a}_{2}} \cos ^{2}(\alpha-\pi / 3)+E_{\mathrm{a}_{3}} \cos ^{2}(\alpha+\pi / 3) . \\
\varepsilon_{\mathrm{y}}=E_{\mathrm{a}_{1}} \sin ^{2} \alpha+E_{\mathrm{a}_{2}} \sin ^{2}(\alpha-\pi / 3)+E_{\mathrm{a}_{3}} \sin ^{2}(\alpha+\pi / 3) .
\end{gathered}
$$

It can readily be shown by equating $\boldsymbol{d} \varepsilon_{\mathrm{x}} / \boldsymbol{d} \alpha$ to zero that $\varepsilon_{i k}$ is diagonal when $x$ and $y$ axes make $a_{0}$ and $\pi / 2-a_{0}$ angles with $\mathbf{a}_{1}$, respectively, and $z$ is along $\mathbf{C}$, where

$$
\alpha_{0}=\frac{1}{2} \operatorname{arctg}\left[\frac{\sqrt{3}\left(E_{\mathrm{a}_{2}}-E_{\mathrm{a}_{3}}\right)}{2 E_{\mathrm{a}_{1}}-E_{\mathrm{a}_{2}}-E_{\mathrm{a}_{3}}}\right] \pm \frac{\pi}{2} k
$$

and $k$ is an integer. In such a coordinate system, the strain field is described by an ellipsoid with $x$ and $y$ being the principal axes [29], while the strain along any particular direction $\mathbf{I}$ is given by a simple sum:

$$
\varepsilon_{1}=\varepsilon_{x} \cos ^{2}(I, x)+\varepsilon_{y} \cos ^{2}(I, y)+\varepsilon_{z} \cos ^{2}(I, z),
$$

where $(\mathbf{I}, \boldsymbol{x})$ denotes the angle between the two directions in the brackets. In other coordinate systems, i.e. when $\alpha \neq \alpha_{0}$, the difference in the quantities defined by Eqs. (A4) and (A1) is given by $\frac{\sqrt{3}}{4}\left(\varepsilon_{\mathrm{a}_{3}}-\varepsilon_{\mathrm{a}_{2}}\right) \sin (2 \alpha)$. Hence, the strains along principal axes of the coordinate system with the $x$ axis along $\mathbf{a}_{1}$ direction, i.e. for $\alpha=\mathbf{0}$, are always correct. 
As we argue in Section 3.4, at relatively high doses the dislocation structure tends to be isotropic in prismatic directions, hence one can assume that $\alpha_{0}=\mathbf{0}$. At low doses and with an anisotropic distribution of prismatic dislocations, such an assumption is, strictly speaking, incorrect, and the exact Eq. (A1) should be used. 


\section{References}

[1] R.A. Holt, J. Nucl. Mater. 159 (1988) 310-338.

[2] R.H. Zee, G.J.C. Carpenter, A. Rogerson, J.F. Walters, J. Nucl. Mater. 150 (1987) 319-330.

[3] S.N. Buckley, Properties of Reactor Materials and Effects of Radiation Damage, ed. W.J. Littler (Butterworths, London, 1962), p. 413.

[4] R.A. Holt, J. Nucl. Mater., 372 (2008) 182-214.

[5] C.H. Woo, U.M. Gösele, J. Nucl. Mater., 119 (1983) 219-228.

[6] C.H. Woo, J. Nucl. Mater., 159 (1988) 237-256.

[7] B. von Guerard, J. Peisl, J. Appl. Cryst., 8 (1975) 161.

[8] S.J. Wooding, L.M. Howe, F. Gao, A.F. Calder, D.J. Bacon, J. Nucl. Mater., 254 (1998) 191-204.

[9] R.E. Stoller, "Primary Radiation Damage Formation," in Comprehensive Nuclear Materials, R. J. M. Konings, T. R. Allen, R. E. Stoller, and S. Yamanaka, Eds, Elsevier Ltd., Amsterdam, 2012, pp. 293-332.

[10] N. De Diego, Y.N. Osetsky, D.J. Bacon, Metallurgical and Materials Transactions A, Physical Metallurgy and Materials Science, 33 (2002) 783-789.

[11] A.V. Barashev, S.I. Golubov, H. Trinkaus, Phil. Mag. A, 81 (2001) 2515-2532.

[12] G.D. Samolyuk, A.V. Barashev, S.I. Golubov, Y.N. Osetsky, R.E. Stoller, Acta Materialia, 78 (2014) p. 173-180.

[13] R.A. Holt, C.H. Woo, C.K. Chow, J. Nucl. Mater., 205 (1993) 293-300.

[14] S.I. Golubov, A.V. Barashev, R.E. Stoller, ORNL/TM-2011/473, Oak Ridge National Laboratory, Oak Ridge, TN, 2011.

[15] S.I. Golubov, A.V. Barashev, R.E. Stoller, B.N. Singh, "Breakthrough in Understanding Radiation Growth of Zirconium," Zirconium in the Nuclear Industry: 17th International Symposium, STP 1543, Robert Comstock and Pierre Barberis, Eds., pp.729-758, doi:10.1520/STP154320130043, ASTM International, West Conshohocken, PA 2014.

[16] S.I. Golubov, B.N. Singh, H. Trinkaus, Phil. Mag. A, 81 (2001) 2533-2552.

[17] A.V. Barashev, S.I. Golubov, Phil. Mag., 89 (2009) 2833-2860.

[18] B.N. Singh, S.I. Golubov, H. Trinkaus, A. Serra, Yu.N. Osetsky, A.V. Barashev, J. Nucl. Mater., 251 (1997) 107-122.

[19] S.I. Golubov, A.V. Barashev, R.E. Stoller, "Mean Field Reaction Rate Theory", in Comprehensive Nuclear Materials, R. J. M. Konings, T. R. Allen, R. E. Stoller, and S. Yamanaka, Eds, Elsevier Ltd., Amsterdam, 2012, pp. 357-391.

[20] M. Griffiths, R.A. Holt, A. Rogerson, J. Nucl. Mater., 225 (1995) 245-258.

[21] R. Risbet, V. Levy, J. Nucl. Mater., 50 (1974) 116-118. 
[22] Y. de Carlan, C. Regnard, M. Griffiths, D. Gilbon, C. Lemaignan, Zirconium in the Nuclear Industry: Eleventh International Symposium, ASTM 1295, E.R. Bradley and G.P. Sabol, Eds., ASTM International, West Conshohocken, PA, 1996, pp. 638-653.

[23] S.I. Golubov, A.M. Ovcharenko, A.V. Barashev, B.N. Singh, Phil. Mag. A, 81 (3) (2001) 643-658.

[24] G.J.C. Carpenter, R.H. Zee, A. Rogerson, J. Nucl. Mater. 159 (1988) 86-100.

[25] A.Jostsons, P.M. Kelly, R.G. Blake, K. Farrell, ASTM-STP 683, (1979) p. 46. [26] S.R. MacEwen, G.J.C. Carpenter, J. Nucl. Mater. 90 (1980) 108-132.

[27] V.N. Shishov, A.V. Nikulina, V.A. Markelov, M.M. Peregud, A.V. Kozlov, S.A. Averin, S.A. Kolbenkov, A.E. Novoselov, Zirconium in the Nuclear Industry: Eleventh International Symposium, ASTM 1295, E.R. Bradley and G.P. Sabol, Eds., ASTM International, West Conshohocken, PA, 1996, pp. 603-622.

[28] D.O. Northwood, V. Fidleris, R.W. Gilbert, G.J.C. Carpenter, J. Nucl. Mater. 61 (196) 123130.

[29] L.D. Landau, E.M. Lifshitz, Theory of Elasticity, $3^{\text {rd }}$ Edition, Course of theoretical physics, Vol. 7, Elsevier Ltd, Amsterdam, 1986. 


\section{Figure captions}

Fig. 1. Cartesian $(x, y)$ coordinate system on the basal plane, where $x$ and $y$ axes make $a$ and $\pi / 2+a$ angles with $\mathbf{a}_{\mathbf{1}}$ direction.

Fig. 2. Dependence of vacancy and interstitial loop number densities on the irradiation dose.

Fig. 3. Comparison of the calculated growth strain with experimental measurements (Figs. 3b and 4a in [24]). Closed and open triangles are for prismatic a strains for iodide and zone-refined purity zirconium, respectively. Open squares show some experimental data on $c$ axis crystals. Fig. 4. Growth strain for the same basic set of parameters (Table 1) as in Fig. 3, but for a wider dose range. Note that the strain values during the break-away stage are similar to those observed experimentally (data taken from Fig. 4a for iodide zirconium in [24]).

Fig. 5. Calculated dose dependence of the sessile loop radii, which reproduce well the experimental values.

Fig. 6. Calculated dose dependence of the sessile loop sink strength.

Fig. 7. Effect of cold work on the growth strain behavior. Solid line is calculated with the basic parameter set (Table 1). Dashed line is calculated with all dislocation densities in the basic parameter set increased by an order of magnitude. Dotted line is calculated with the hindered times higher dislocation densities. Dash-dotted line is calculated with $\rho_{\mathbf{d}}^{\mathbf{a}} / \rho_{\mathbf{d}}^{\mathbf{c}}=\mathbf{1 0}$, i.e. two times higher ratio of $a$ to $c$ dislocation densities.

Fig. 8. Dose dependence of growth strain behavior up to hundred dpa, for high (dotted line) and low (solid line) densities of prismatic dislocations. Note that, at high doses, the strain behavior is independent of the initial dislocation density.

Fig. 9. Dose dependence of loop radii calculated for $\rho_{\mathrm{d}}^{\mathrm{a}}=3 \times 10^{12} \mathrm{~m}^{-2}$.

Fig. 10. Dose dependence of the loop sink strengths for $\rho_{\mathrm{d}}^{\mathrm{a}}=3 \times 10^{12} \mathrm{~m}^{-2}$. Note that, at high irradiation doses, the loop sink strength is much higher than the dislocation density.

Fig. 11. Decrease of growth strain with increasing ratio of $a$ - to $c$-loop densities. Note an enhanced radiation resistance of $\mathrm{Zr}$ due to suppression of c-type loop nucleation.

Fig. 12. Dose dependence of sessile interstitial and vacancy loop radii calculated for the case in which the initial density of $\mathbf{a}_{1}$ dislocations is six times higher than in the other two directions. Note co-existence of vacancy- and interstitial-type prismatic loops for a limited, several dpa, period of irradiation. 
Fig. 13. Same as in Fig. 12 but for growth strain. Note that the strain along the direction where vacancy loops grow and, then, positive, when vacancy loops dissolved.

Fig. 14. Dose dependence of growth strain for the same case as in Fig. 13 (designated as low dislocation density), and for the case in which all dislocation densities are increased by an order of magnitude (designated as high dislocation density).

Fig. 15. Same as in Fig. 14 designated as high dislocation density but for the loop radii.

Fig. 16. Dose dependence of growth strain for the case when the initial density of $\mathbf{a}_{1}$ dislocations is ten time lower than in the other two $a$ directions. Note that in this case vacancy loops initially grow and then dissolve in two $a$ directions rather than one $a$ direction, as in Figs. 14 and 15.

Fig. 17. Same as in Fig. 16 but for the loop radii. 
Table 1. Basic set of model parameters used in calculations unless stated otherwise.

\begin{tabular}{|c|c|c|}
\hline Definition & Symbol & Value \\
\hline Fraction of defects recombining in cascades & $\varepsilon_{\mathrm{r}}$ & 0.9 \\
\hline Fraction of SIAs produced in mobile clusters & $\varepsilon_{\mathrm{i}}^{\mathrm{g}}$ & 0.2 \\
\hline $\begin{array}{l}\text { Terminal density of } a \text {-type vacancy- and interstitial- } \\
\text { type sessile loops }\end{array}$ & $N_{v}^{a, \max }, N_{\mathrm{i}}^{a, \max }$ & $10^{22} \mathrm{~m}^{-3}$ \\
\hline Terminal dose for nucleation of sessile $a$ loops & $\phi_{\max }^{\mathrm{a}}$ & $3.84 \mathrm{dpa}$ \\
\hline Terminal density of $c$-type sessile vacancy loops & $N^{c, \max }$ & $10^{21} \mathrm{~m}^{-3}$ \\
\hline Starting dose for nucleation of $c$ vacancy loops & $\phi_{0}^{\mathrm{c}}$ & $3 \mathrm{dpa}$ \\
\hline Terminal dose for nucleation of $c$ vacancy loops & $\phi_{\max }^{\mathrm{c}}$ & 23 dpa \\
\hline Nucleation parameter for $c$ loops in Eq. (28) & $A$ & 5 \\
\hline Burgers vector of $a$ loops & $b_{\mathrm{a}}$ & $3 \times 10^{-10} \mathrm{~m}$ \\
\hline Burgers vector of $c$ loops & $b_{\mathrm{c}}$ & $5 \times 10^{-10} \mathrm{~m}$ \\
\hline $\begin{array}{l}\text { Capture radii of dislocations, and sessile vacancy and } \\
\text { interstitial prismatic loops for SIA clusters }\end{array}$ & $r_{\mathrm{cd}}$ & $6 \times 10^{-10} \mathrm{~m}$ \\
\hline Densities of $a_{1}, a_{2}, a_{3}$ dislocations & $\rho_{\mathrm{d}}^{\mathrm{a}_{1}}, \rho_{\mathrm{d}}^{\mathrm{a}_{2}}, \rho_{\mathrm{d}}^{\mathrm{a}_{3}}$ & $10^{12} \mathrm{~m}^{-2}$ \\
\hline Density of $c$ dislocations & $\rho_{\mathbf{d}}^{\mathbf{c}}$ & $0.6 \times 10^{12} \mathrm{~m}^{-2}$ \\
\hline $\begin{array}{l}\text { Neutron fluence corresponding to } 1 \mathrm{dpa} \text { of the NRT } \\
\text { standard dose }\end{array}$ & --- & $10^{25} \mathrm{n} / \mathrm{m}^{2}$ \\
\hline
\end{tabular}


Figure

Fig. 1.

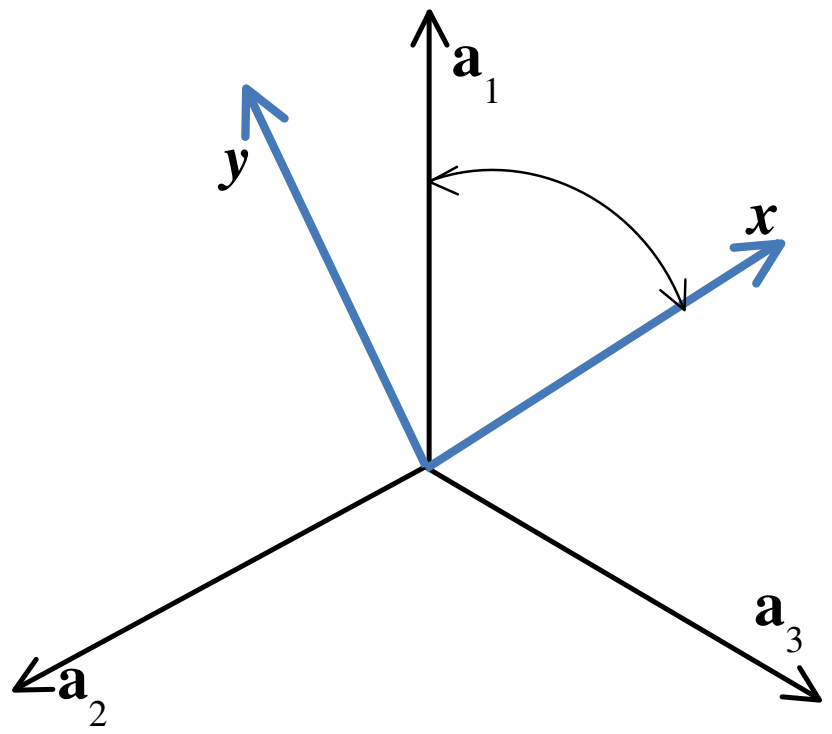


Fig. 2.

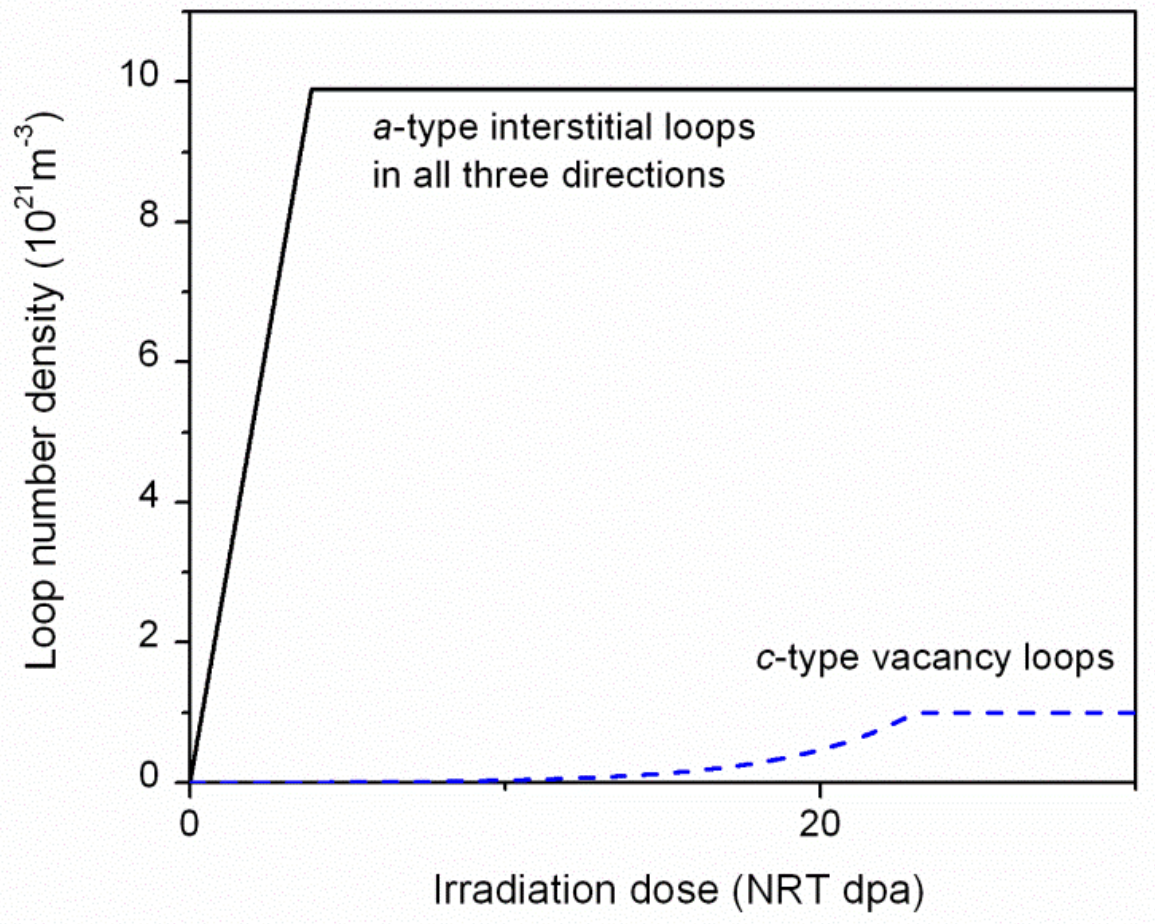


Fig. 3.

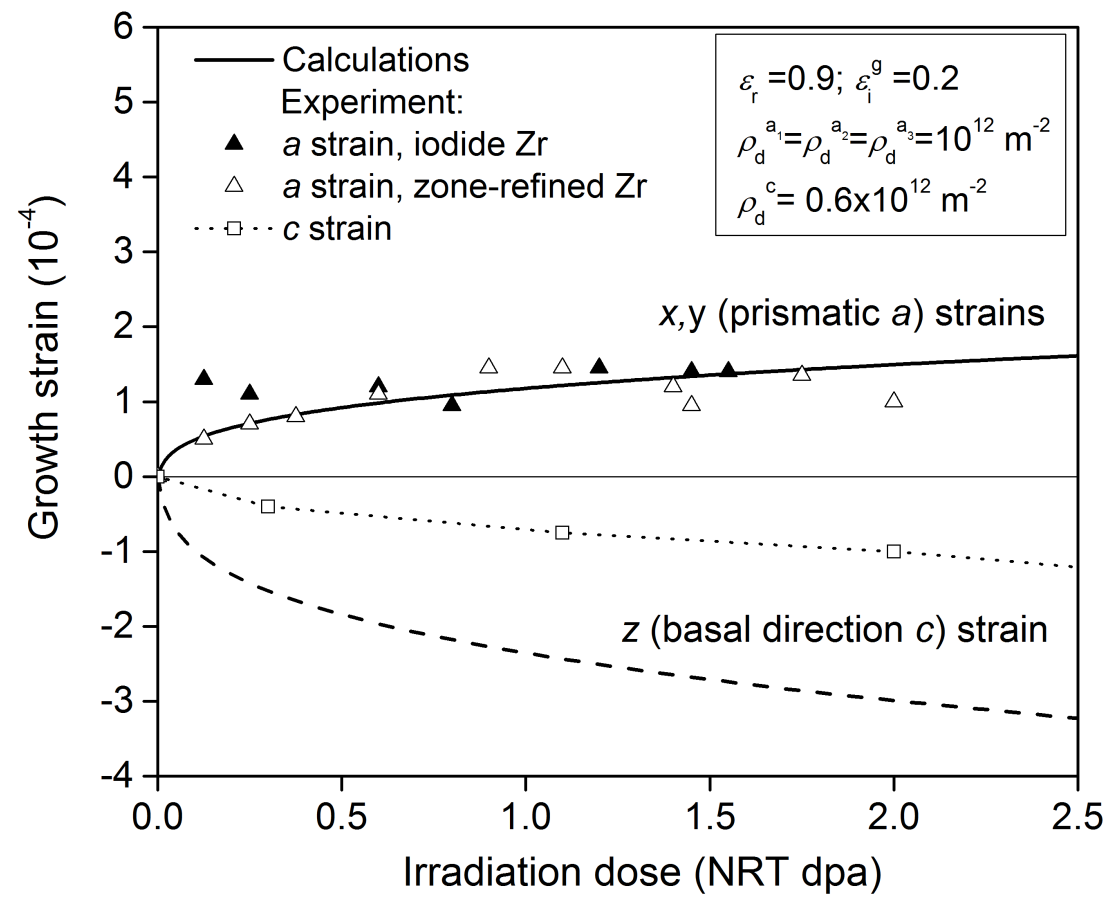


Fig. 4.

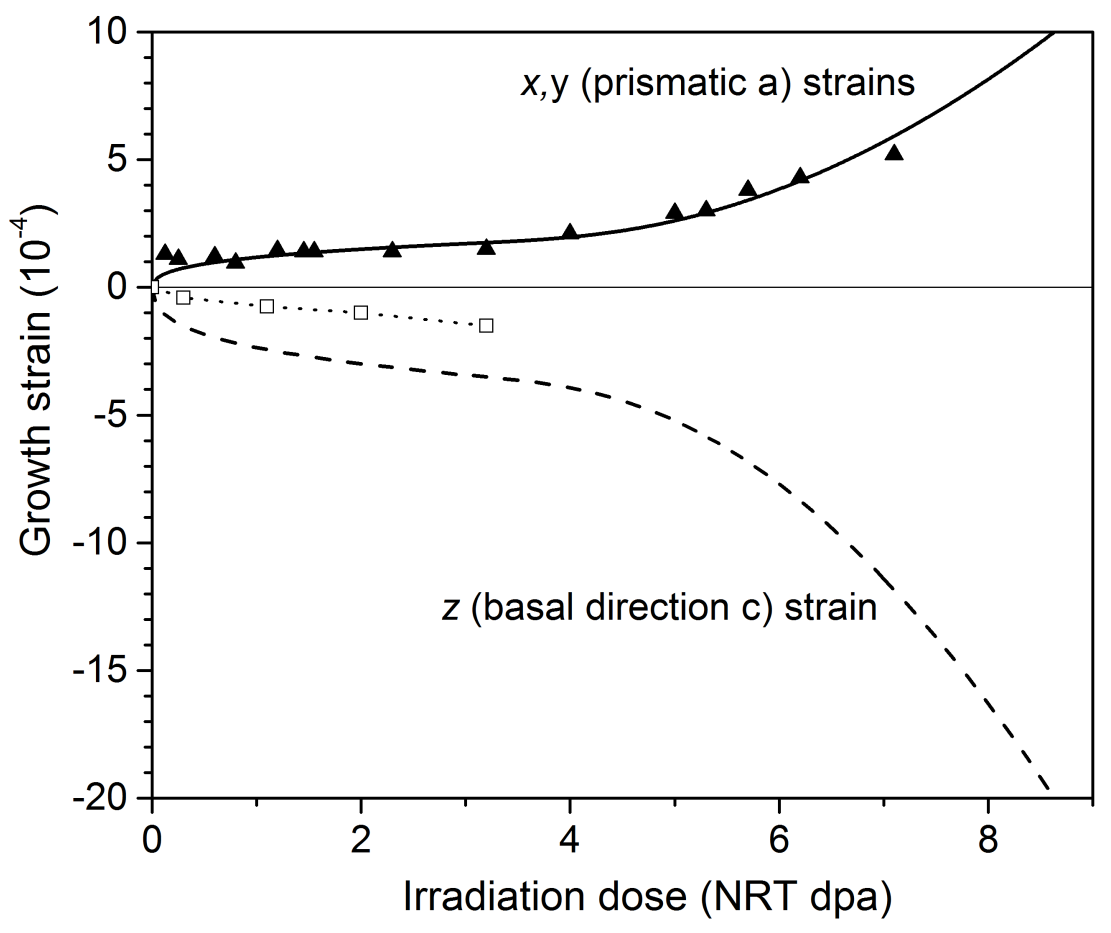


Fig. 5.

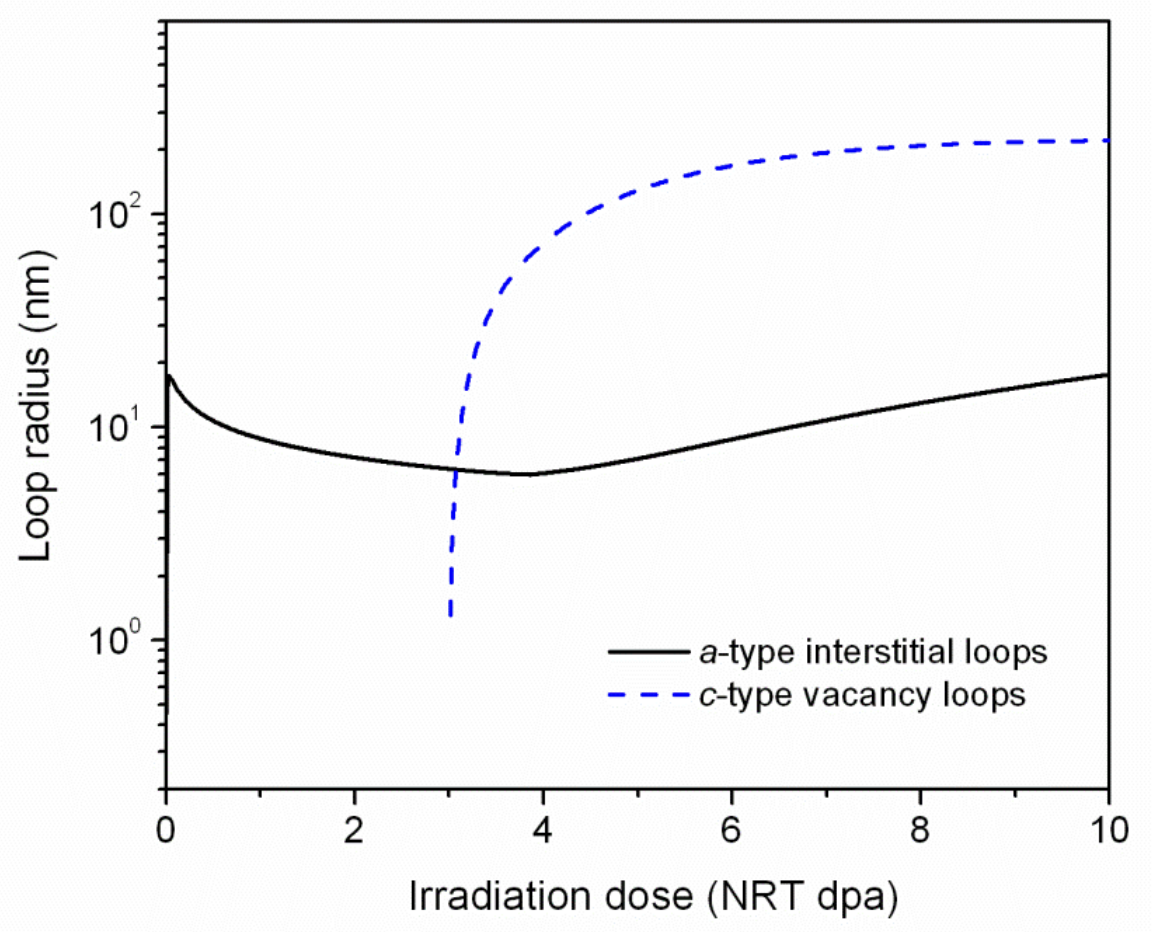


Fig. 6.

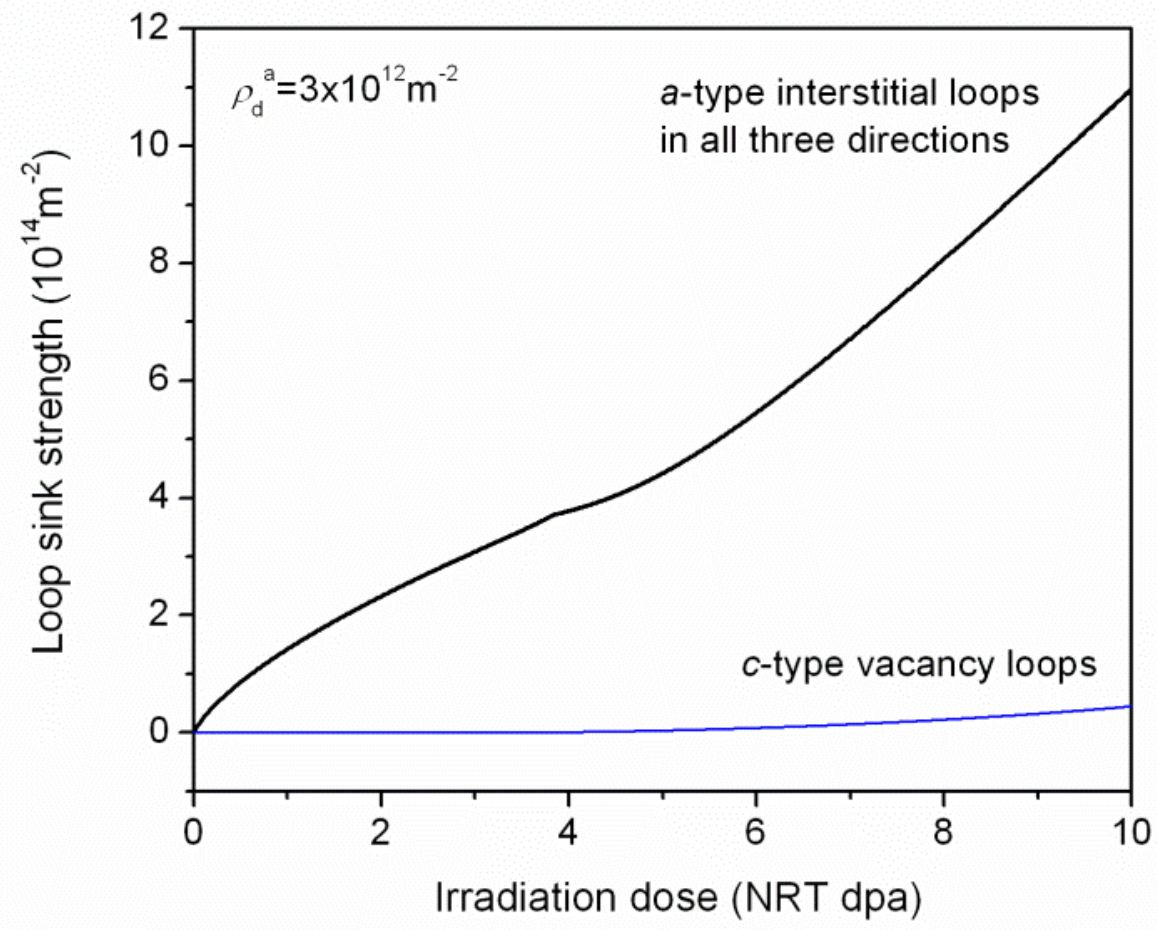


Fig. 7.

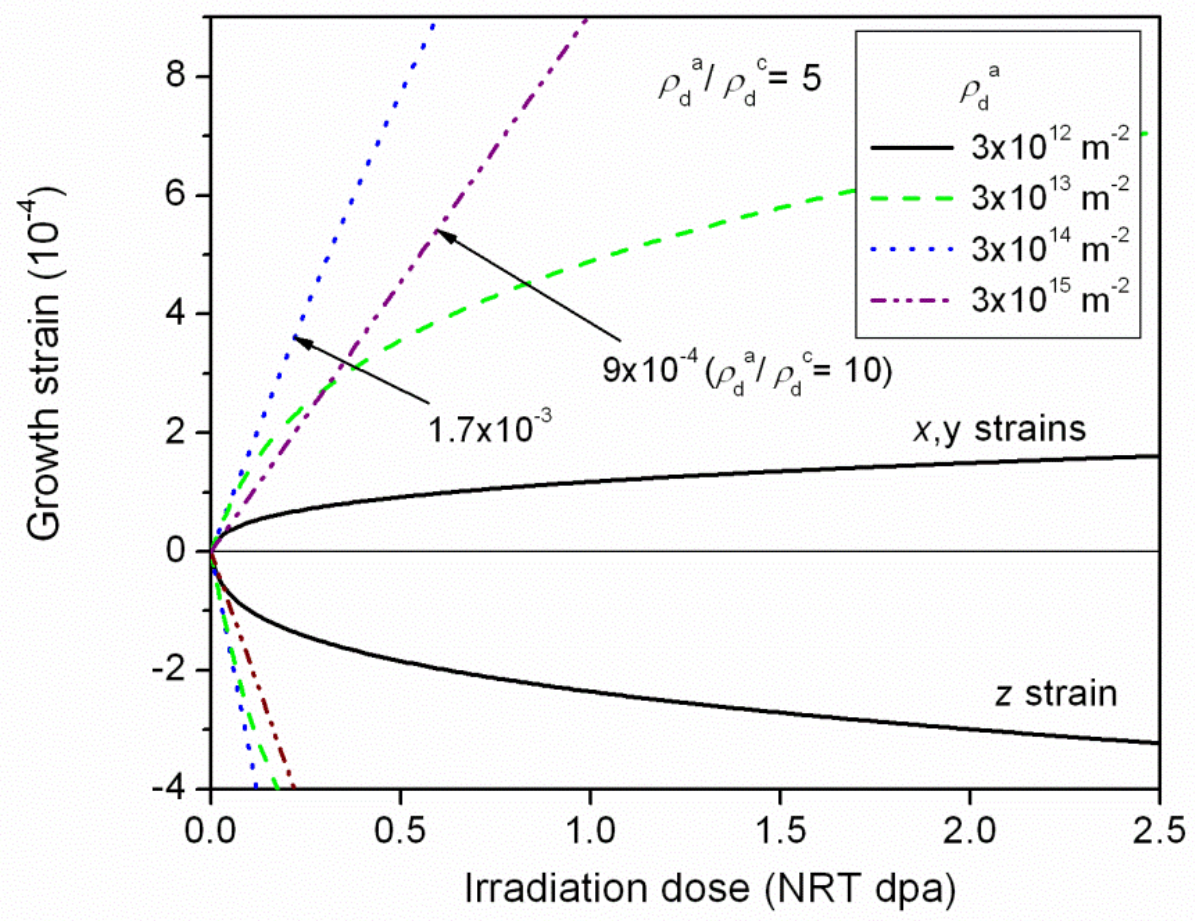


Fig. 8.

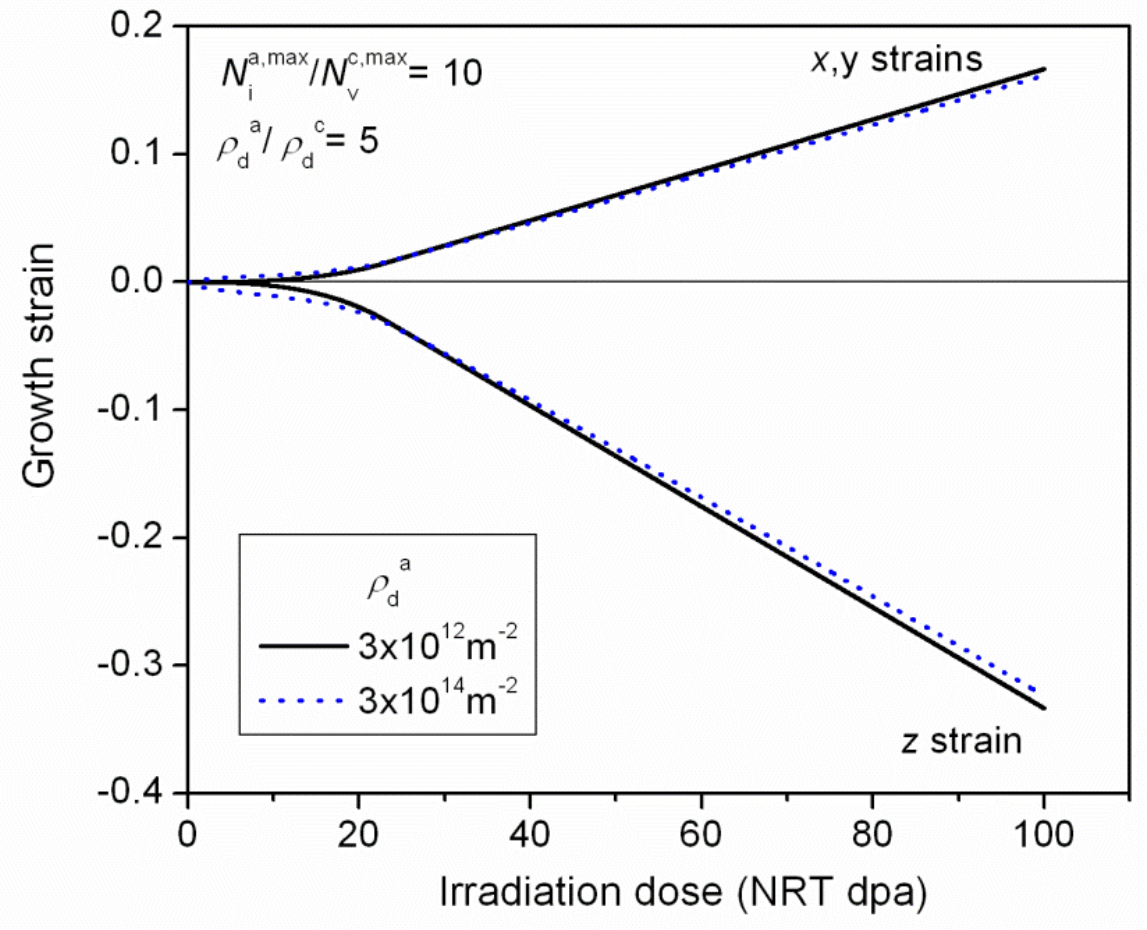


Fig. 9.

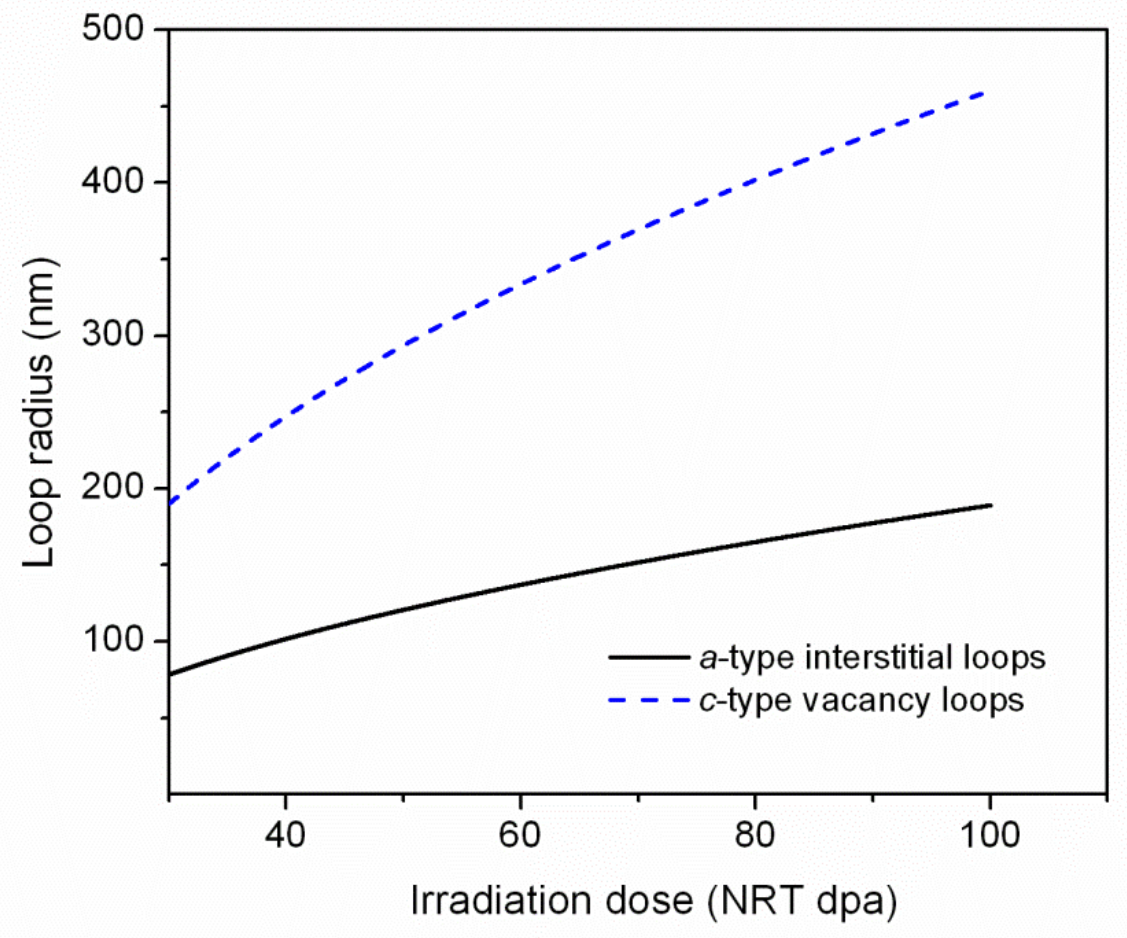


Fig. 10.

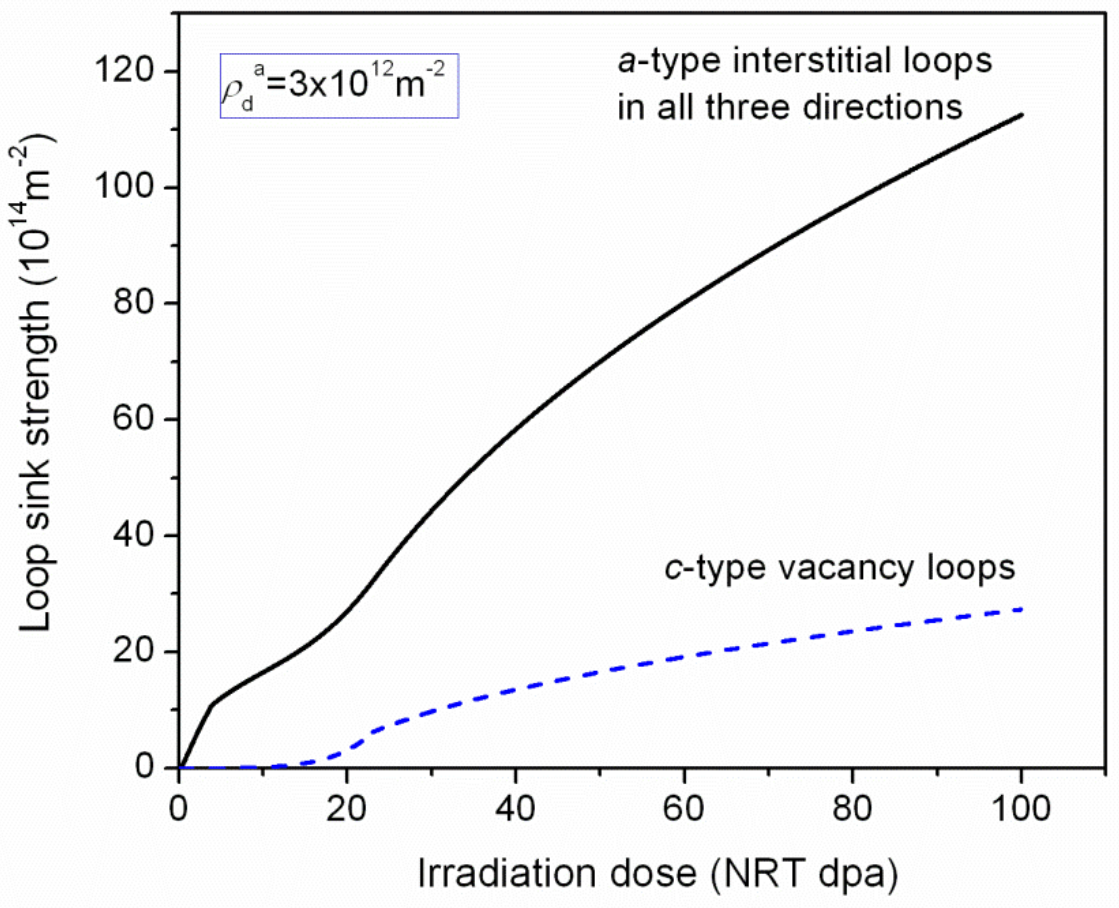


Fig. 11.

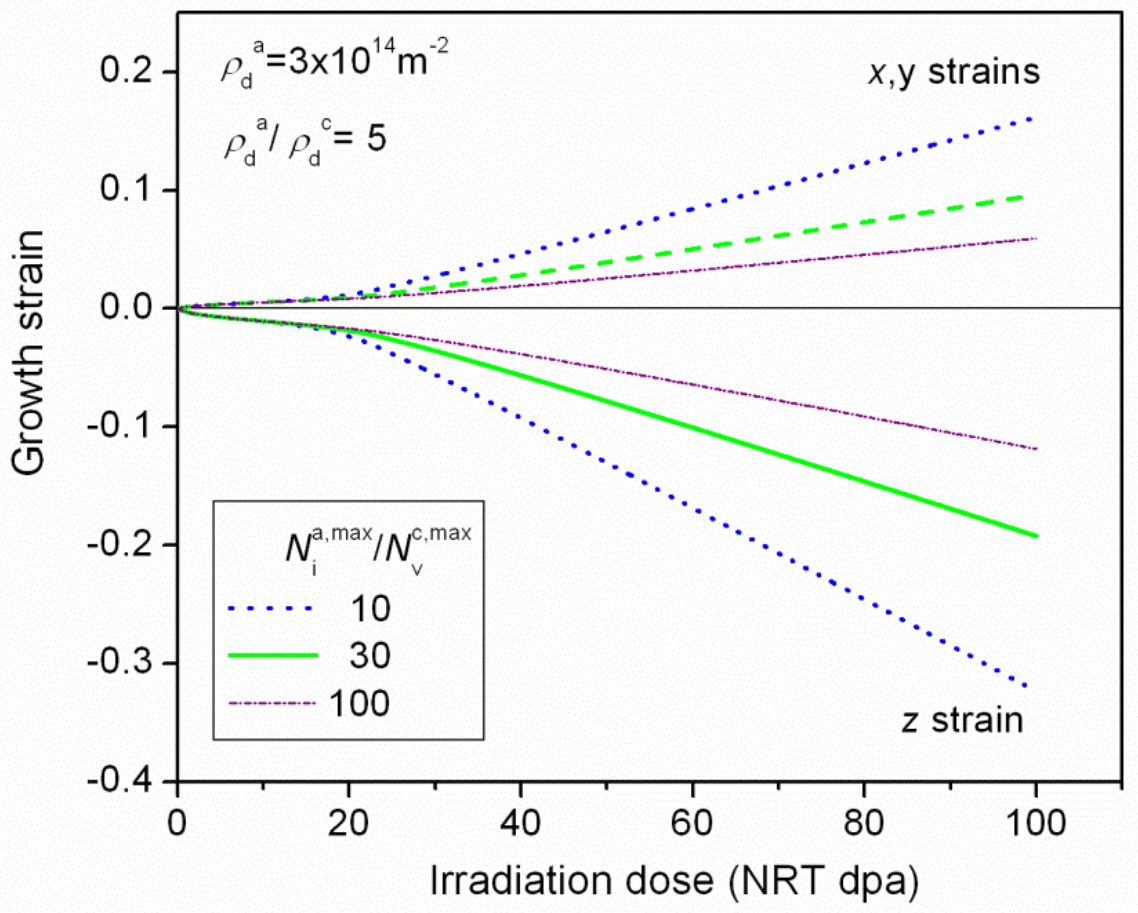


Fig. 12.

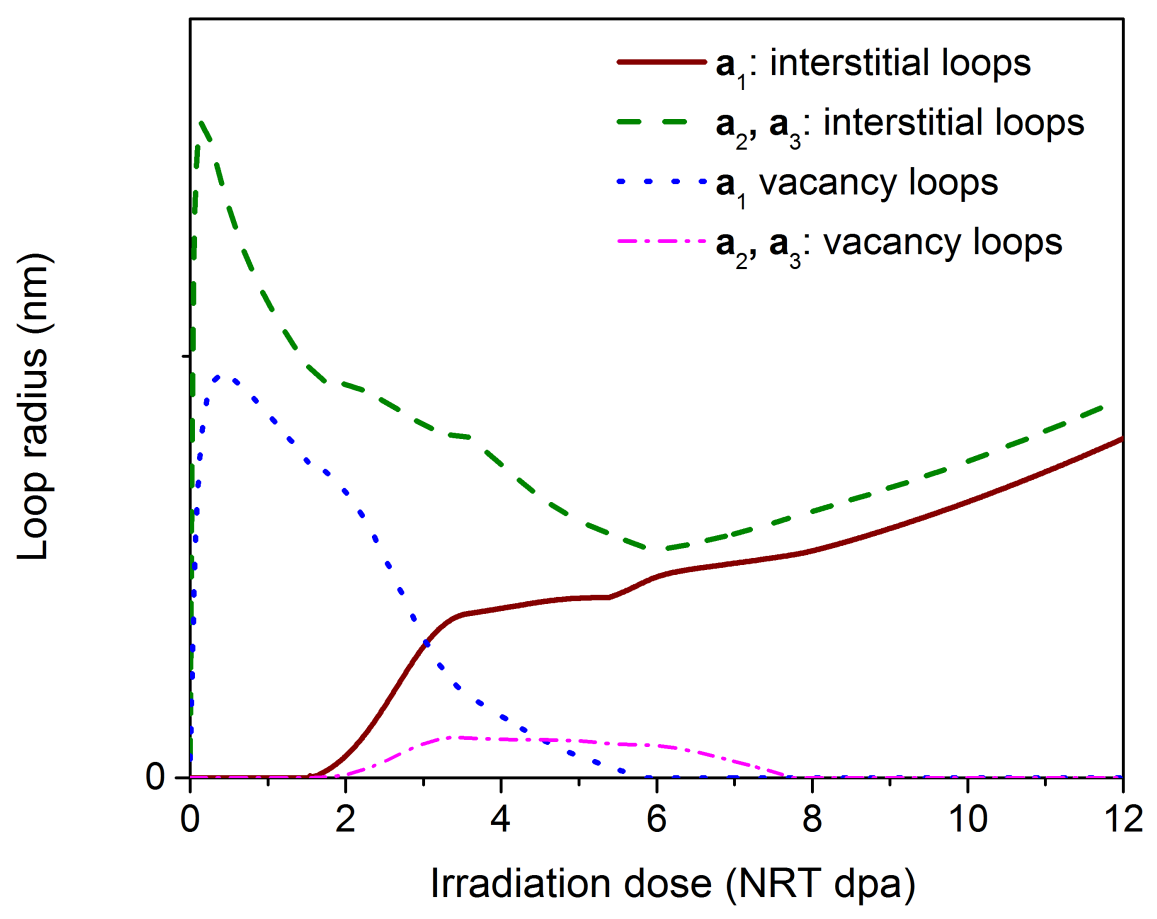


Fig. 13.

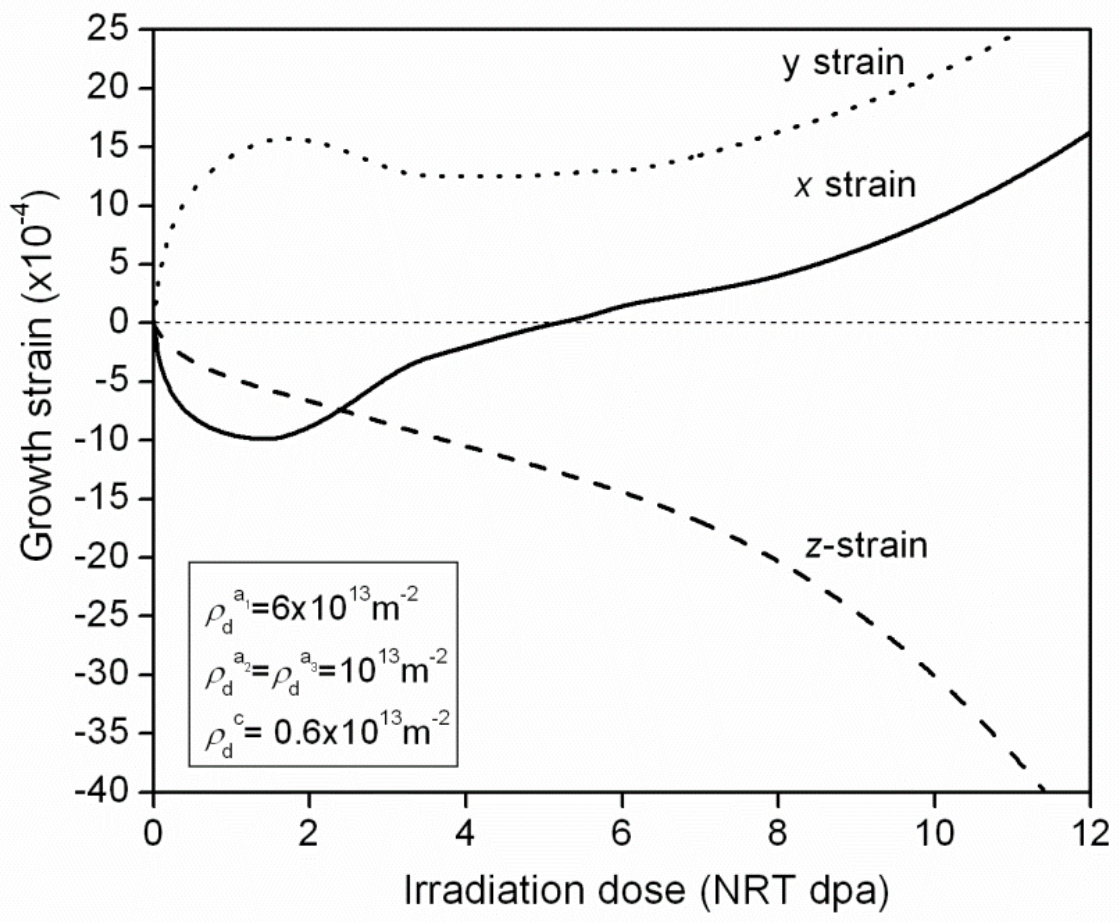


Fig. 14.

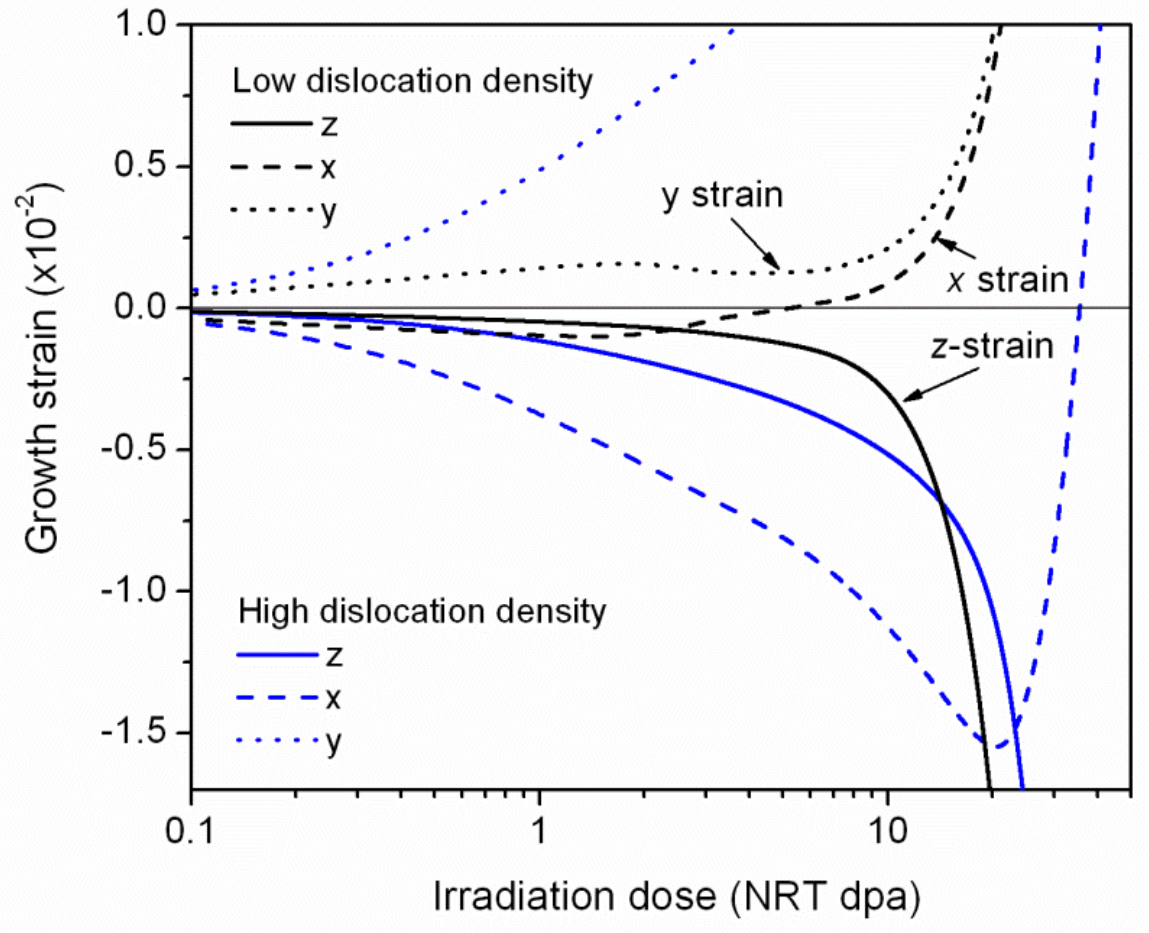


Fig. 15.

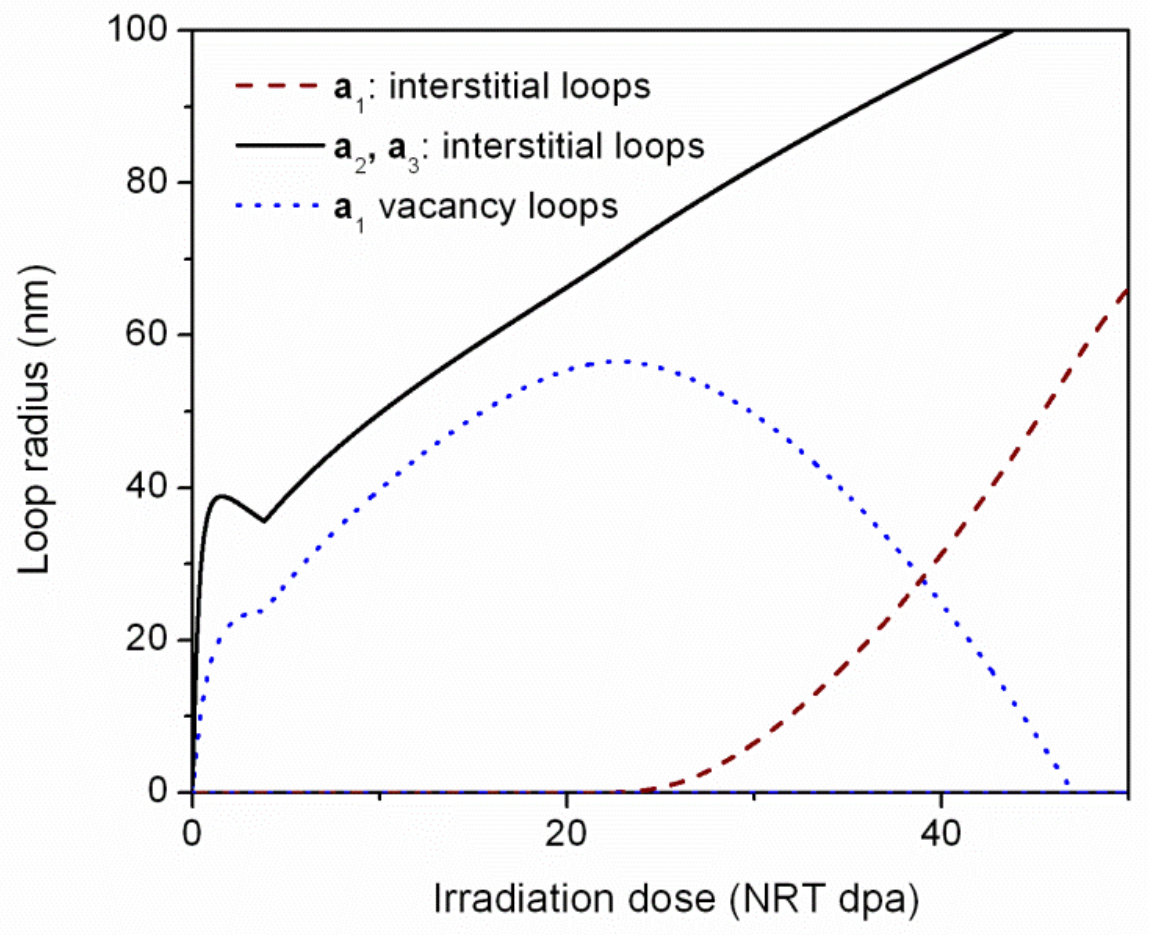


Fig. 16.

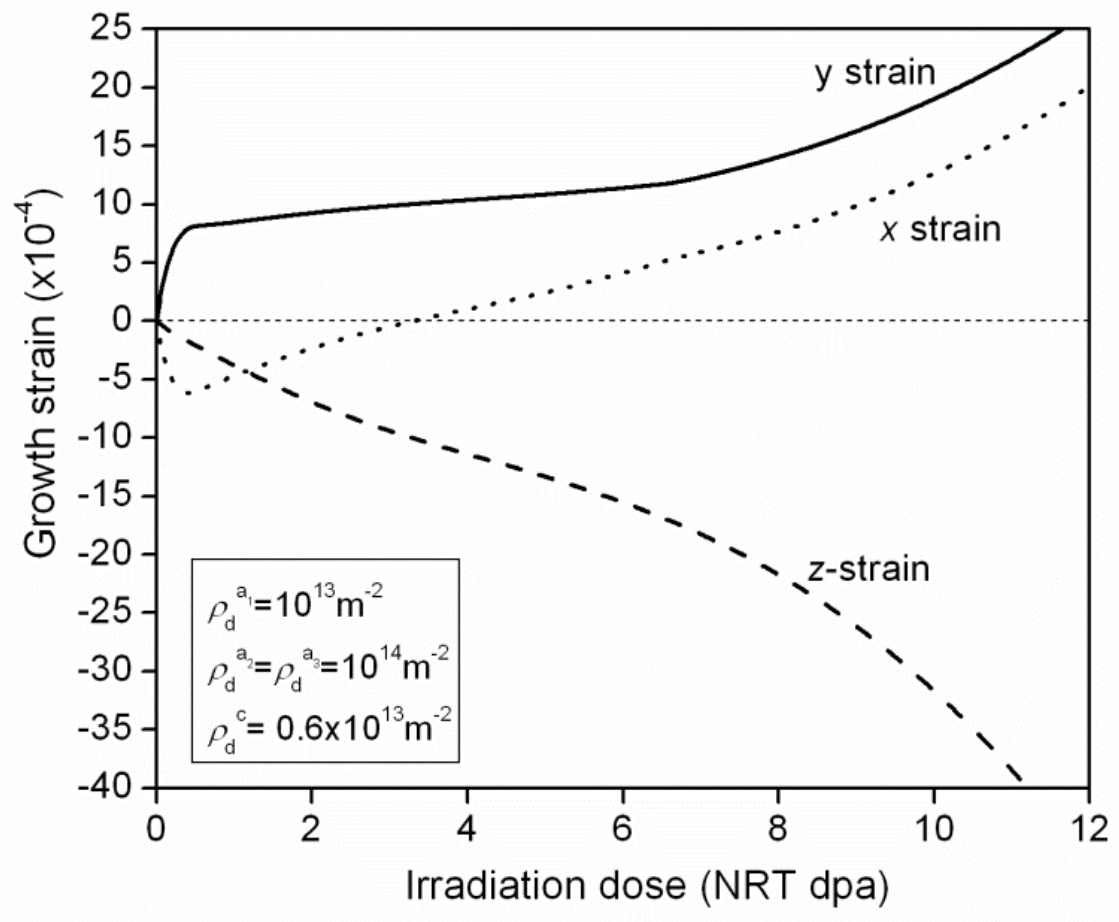


Fig. 17.

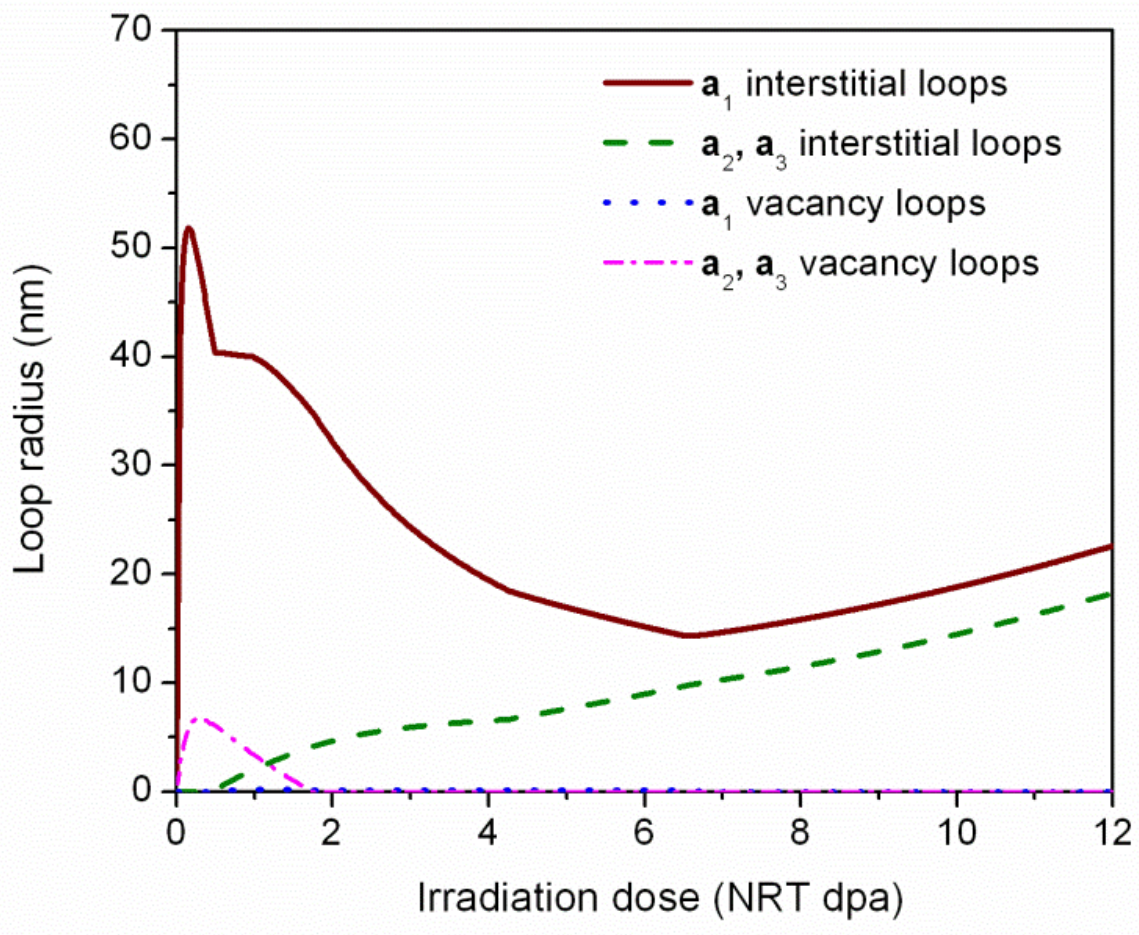

